\title{
Orbital Stability of Solitary Waves for Generalized Symmetric Regularized-Long-Wave Equations with Two Nonlinear Terms
}

\author{
Weiguo Zhang, ${ }^{1}$ Xu Chen, ${ }^{1}$ Zhengming Li, ${ }^{2}$ and Haiyan Zhang ${ }^{1}$ \\ ${ }^{1}$ College of Science, University of Shanghai for Science and Technology, Shanghai 200093, China \\ ${ }^{2}$ Business School, University of Shanghai for Science and Technology, Shanghai 200093, China \\ Correspondence should be addressed to Weiguo Zhang; zwgzwm@126.com
}

Received 28 February 2014; Accepted 8 May 2014; Published 26 May 2014

Academic Editor: Wan-Tong Li

Copyright (c) 2014 Weiguo Zhang et al. This is an open access article distributed under the Creative Commons Attribution License, which permits unrestricted use, distribution, and reproduction in any medium, provided the original work is properly cited.

\begin{abstract}
This paper investigates the orbital stability of solitary waves for the generalized symmetric regularized-long-wave equations with two nonlinear terms and analyzes the influence of the interaction between two nonlinear terms on the orbital stability. Since $J$ is not onto, Grillakis-Shatah-Strauss theory cannot be applied on the system directly. We overcome this difficulty and obtain the general conclusion on orbital stability of solitary waves in this paper. Then, according to two exact solitary waves of the equations, we deduce the explicit expression of discrimination $d^{\prime \prime}(c)$ and give several sufficient conditions which can be used to judge the orbital stability and instability for the two solitary waves. Furthermore, we analyze the influence of the interaction between two nonlinear terms of the equations on the wave speed interval which makes the solitary waves stable.
\end{abstract}

\section{Introduction}

Symmetric regularized-long-wave equations (SRLWE)

$$
\begin{gathered}
u_{x x t}-u_{t}=\left(v+\frac{1}{2} u^{2}\right)_{x}, \\
v_{t}+u_{x}=0,
\end{gathered}
$$

which are the mathematical models describing the propagation of weakly nonlinear ion acoustic waves [1] and the typical equations in the field of nonlinear science, arise in many other areas of nonlinear mathematical physics [2]. References $[1,2]$ studied the solitary wave solutions, conservation laws, and interaction among the solitary wave solutions of (1). Moreover, [3-5] discussed the global solution and numerical solution of (1).

Many authors have studied some extended forms of (1).

Guo [3] studied the periodic initial value problem for generalized nonlinear wave equations including (1)

$$
\begin{gathered}
u_{t}-u_{x x t}+\rho_{x}+f(u)_{x}=g\left(u, \rho, u_{x}\right), \\
\rho_{t}+u_{x}=h(\rho),
\end{gathered}
$$

by spectral method, then proved the existence and uniqueness of the global generalized solution and classical solution, and gave the convergence and error estimates for the approximate solution in 1987. Zhang [6] obtained the exact solitary wave solutions for a class of the generalized SRLWE with high-order nonlinear terms in 2003.

In terms of the orbital stability of solitary wave solutions, Chen [7] studied it in 1998 for the following generalized SRLWE:

$$
u_{t t}-u_{x x}+f(u)_{x t}-u_{x x t t}=0
$$

where $f(u)$ is a $C^{1}$ function, satisfying $f(s)>0$ if $s>$ 0 and $|f(s)|=o\left(|s|^{p}\right) ;\left|f^{\prime}(s)\right|=o\left(|s|^{p-1}\right)$ as $s \rightarrow 0$ for $p>1$. In particular, $\varphi_{c}>0$ in the solitary wave solution $\left(\varphi_{c}, \psi_{c}\right)^{T}$ (T represents transposition) in Assumption 1 of [7]. Moreover, $H_{c}$ only has a simple negative eigenvalue, whose kernel is spanned by $\varphi_{c}^{\prime}$. In addition, the rest of its eigenvalues are positive and bounded away from zero. 
In this paper, we will consider the orbital stability and instability of solitary wave solutions for the following generalized SRLWE with two nonlinear terms:

$$
\begin{gathered}
u_{x x t}-u_{t}=\left(v+b_{2} u^{2}+b_{3} u^{3}\right)_{x} \\
b_{i}=\text { constant, } \quad b_{3} \geq 0, \quad i=2,3, \\
v_{t}+u_{x}=0 .
\end{gathered}
$$

Our purpose is to investigate the influence of the interaction of the nonlinear terms on the orbital stability.

Equation (4) is the generalization of (1). If (4) is converted into (3), then $f(u)=b_{2} u^{2}+b_{3} u^{3}$, where $f(u)$ has two nonlinear terms and the symbols of $b_{2}, b_{3}$ are unfixed. Indeed, $f(u)$ is not always positive when $u>0$, so the problem studied in this paper is not included by [7]. In the other hand, according to Theorem 1 in this paper, (4) has two bell-profile solitary wave solutions $\left(\varphi_{i}, \psi_{i}\right)^{T}, i=1,2$, where $\varphi_{1}(\xi)>0$ and $\varphi_{2}(\xi)<0$. But the orbital stability of the solitary wave solution $\left(\varphi_{2}(\xi), \psi_{2}(\xi)\right)^{T}$ is not considered in [7]. In this paper, we will consider it as well. So the content of this study is new. More significantly, we will study the influence of the interaction between nonlinear terms $b_{2} u^{2}$ and $b_{3} u^{3}$ on the orbital stability. It is meaningful for the stability in the application of the practical problems and the selection of the models.

The paper is organized as follows. In Section 2, we will present two exact bell-profile solitary wave solutions of (4) and local existence for the solution of Cauchy problem. In Section 3, we will verify that (4) and its solitary wave solutions meet the requirements of the orbital stability theory of Grillakis-Shatah-Strauss and give the general conclusion. In Section 4, according to two exact solitary waves of the equations obtained in Section 2, we deduce the explicit expression of discrimination $d^{\prime \prime}(c)$ and give several sufficient conditions which can be used to judge the orbital stability and instability for the two solitary waves. Moreover, we will analyze the influence of two nonlinear terms on the orbital stability. In Section 5, we will focus on studying the orbital instability of solitary wave solutions for (4). Since the skew symmetric operator $J$ is not onto, we will define a new conservational functional $I(\vec{u})=\int_{R} \vec{u} d x$ and estimate solutions of the initial value problem. We will construct a formal Lyapunov function and present the sufficient condition on orbital instability of solitary wave solutions.

\section{The Bell-Profile Solitary Wave Solutions and Local Existence for the Solution of Cauchy Problem}

According to [6], the solitary wave solution of (4) satisfies

$$
\begin{gathered}
u(x, t)=u(\xi)=u(x-c t) \\
v(x, t)=v(\xi)=v(x-c t)
\end{gathered}
$$

$$
\begin{gathered}
-c u^{\prime \prime \prime}(\xi)+c u^{\prime}(\xi)=v^{\prime}(\xi)+\left(b_{2} u^{2}(\xi)+b_{3} u^{3}(\xi)\right)_{\xi} \\
v^{\prime}(\xi)=\frac{1}{c} u^{\prime}(\xi)
\end{gathered}
$$

where $u^{\prime}(\xi), u^{\prime \prime}(\xi) \rightarrow 0,|\xi| \rightarrow \infty$. Their exact expressions are given by the following theorem.

Theorem 1. Suppose that $c^{2}-1>0$.

(1) If $b_{3} c>0$, or $b_{3}=0$ and $b_{2} c>0$, then (4) has a bellprofile solitary wave solution

$$
\begin{gathered}
u(x, t)=\varphi_{1}(\xi)=\varphi_{1}(x-c t), \\
v(x, t)=\psi_{1}(\xi)=\frac{1}{c} \varphi_{1}(x-c t),
\end{gathered}
$$

where

$$
\begin{gathered}
\varphi_{1}(\xi)=\frac{A_{1} \operatorname{sech}^{2}\left(\alpha_{1} / 2\right) \xi}{2+B_{1} \operatorname{sech}^{2}\left(\alpha_{1} / 2\right) \xi} \\
\alpha_{1}=\frac{\sqrt{c^{2}-1}}{c}, \quad A_{1}=\frac{3 \sqrt{2}\left(c^{2}-1\right)}{\sqrt{c\left[2 b_{2}^{2} c+9 b_{3}\left(c^{2}-1\right)\right]}}, \\
B_{1}=-1+\frac{b_{2} A_{1}}{3 \alpha_{1}^{2} c}
\end{gathered}
$$

(2) If $b_{3} c>0$, or $b_{3}=0$ and $b_{2} c<0$, then (4) has another bell-profile solitary wave solution

$$
\begin{gathered}
u(x, t)=\varphi_{2}(\xi)=\varphi_{2}(x-c t), \\
v(x, t)=\psi_{2}(\xi)=\frac{1}{c} \varphi_{2}(x-c t),
\end{gathered}
$$

where

$$
\begin{gathered}
\varphi_{2}(\xi)=\frac{A_{2} \operatorname{sech}^{2}\left(\alpha_{2} / 2\right) \xi}{2+B_{2} \operatorname{sech}^{2}\left(\alpha_{2} / 2\right) \xi}, \\
\alpha_{2}=\alpha_{1}, \quad A_{2}=-A_{1}, \quad B_{2}=-1+\frac{b_{2} A_{2}}{3 \alpha_{2}^{2} c}=-2-B_{1} .
\end{gathered}
$$

Next, we study the local existence for the solution of Cauchy problem for (4) by semigroup theory. Firstly, we give two lemmas (see $[8,9])$.

Lemma 2 (Hille-Yosida-Phillips). A linear unbounded operator $A$ is the infinitesimal generator of a $C_{0}$ semigroup of $\{T(t)$ : $t \geq 0\}$ if and only if $A$ is a closed operator with dense domain and there exist real numbers $M$ and $\omega$, such that when $\lambda>\omega$, one has 
(1) $\lambda \in \rho(A)$,

(2) $\left\|R(\lambda ; A)^{n}\right\| \leq M /(\lambda-\omega)^{n}, n=1,2, \ldots$,

where $\rho(A)$ is the resolvent set and $R(\lambda ; A)^{n}$ is the resolvent of A.

Lemma 3. Consider the Cauchy problem of nonlinear equation

$$
\begin{gathered}
\frac{d u(t)}{d t}=A u(t)+f(t, u(t)), \quad t>0, \\
u(0)=u_{0}, \quad u_{0} \in X .
\end{gathered}
$$

If the following two conditions hold:

(1) $A$ is the infinitesimal generator of a $C_{0}$ semigroup $T(t)$ in $X$;

(2) $f \in C\left(R^{+} \times X, X\right)$ satisfies the Lipschitz manner, which means for any $T>0$,

there exists $K=K(t)$, such that $\|f(t, u)-f(t, v)\| \leq K(t) \| u-$ $v \|$, for all $u, v \in X, t \in[0, T]$, then the initial value problem (8) has a unique solution

$$
u(t)=T(t) u_{0}+\int_{0}^{t} T(t-s) f(s, u(s)) d s
$$

in $R^{+}$.

From Lemmas 2 and 3, we can prove the following Lemma 4, which describes the local existence for the solution of Cauchy problem for (4).

Lemma 4. For any $\vec{u}_{0} \in X\left(H^{1}(R) \times L^{2}(R)\right)$, there exists $t_{0}>$ 0 , which only depends on $\left\|\vec{u}_{0}\right\|_{X}$, such that (4) has a unique solution $\vec{u} \in C\left(\left[0, t_{0}\right) ; H^{1}(R) \times L^{2}(R)\right)$ with $\vec{u}(0)=\vec{u}_{0}$.

Proof. Firstly, (4) can be written as

$$
\begin{gathered}
u_{t}=(\Delta-1)^{-1}\left(v+b_{2} u^{2}+b_{3} u^{3}\right)_{x}, \\
b_{i}=\text { constant }, \quad b_{3} \geq 0, \\
v_{t}=-u_{x},
\end{gathered}
$$

where $\Delta=\partial^{2} / \partial x^{2} \cdot(\Delta-1)^{-1}$ is the pseudodifferential operator. The initial value problem of (10) is equal to

$$
\begin{gathered}
\frac{d \vec{u}(t)}{d t}=A \vec{u}(t)+f(t, \vec{u}(t)), \quad t>0, \\
\vec{u}(0)=\vec{u}_{0},
\end{gathered}
$$

where

$$
\begin{gathered}
\vec{u}(t)=\left(\begin{array}{c}
u(t) \\
v(t)
\end{array}\right), \quad \vec{u}_{0}=\left(\begin{array}{l}
u(0) \\
v(0)
\end{array}\right), \\
A=\left(\begin{array}{cc}
0 & (\Delta-1)^{-1} \partial_{x} \\
-\partial_{x} & 0
\end{array}\right), \\
f(t, \vec{u}(t))=\left(\begin{array}{c}
(\Delta-1)^{-1}\left(b_{2} u^{2}+b_{3} u^{3}\right)_{x} \\
0
\end{array}\right) .
\end{gathered}
$$

Since for any $T>0$, there exists $K=K(t)$, such that for any $\vec{u}_{1}, \vec{u}_{2} \in X, t \in[0, T]$, we have

$$
\begin{aligned}
&\left\|f\left(t, \vec{u}_{1}\right)-f\left(t, \vec{u}_{2}\right)\right\| \\
&= \|\left(\begin{array}{c}
\left.(\Delta-1)^{-1}\left(b_{2} u_{1}^{2}+b_{3} u_{1}^{3}\right)_{x}\right) \\
0
\end{array}\right) \\
&-\left(\begin{array}{c}
\left.(\Delta-1)^{-1}\left(b_{2} u_{2}^{2}+b_{3} u_{2}^{3}\right)_{x}\right) \\
0
\end{array}\right) \| \\
&= \|\left(\begin{array}{c}
(\Delta-1)^{-1}\left[b_{2}\left(\begin{array}{c}
\left.\left.u_{1}^{2}-u_{2}^{2}\right)+b_{3}\left(u_{1}^{3}-u_{2}^{3}\right)\right]_{x} \\
0
\end{array}\right) \|\right. \\
\leq K(t)\left\|\vec{u}_{1}-\vec{u}_{2}\right\| .
\end{array}\right.
\end{aligned}
$$

Therefore, $f(t, \vec{u}(t))$ satisfies the local Lipschitz manner.

Now we want to verify that $A$ is the infinitesimal generator of a $C_{0}$ semigroup in $X$ and $D(A)=H^{1} \times L^{2}$.

According to Lemma 2, we only need to prove that there exists $\omega$, such that

$$
\left\|(\lambda I-A)^{-1}\right\| \leq \frac{1}{\lambda-\omega}
$$

if $\lambda>\omega$ and $\lambda \in \rho(A)$.

Indeed, since $\lambda \in \rho(A)$, for any $\vec{v}=\left(\begin{array}{c}\vec{v}_{1} \\ \vec{v}_{2}\end{array}\right) \in X$, we have $\vec{u}=\left(\begin{array}{l}\vec{u}_{1} \\ \vec{u}_{2}\end{array}\right) \in D(A)$ and $(\lambda I-A) \vec{u}=\vec{v}$. Thus $\vec{u}=(\lambda I-A)^{-1} \vec{v}$. Taking the Fourier transform yields

$$
\begin{aligned}
& \widehat{u}_{1}=\frac{\lambda}{\lambda^{2}+\xi^{2} /\left(\xi^{2}+1\right)} \widehat{v}_{1}-\frac{i \xi /\left(1+\xi^{2}\right)}{\lambda^{2}+\xi^{2} /\left(1+\xi^{2}\right)} \widehat{v}_{2}, \\
& \widehat{u}_{2}=\frac{\lambda}{\lambda^{2}+\xi^{2} /\left(\xi^{2}+1\right)} \widehat{v}_{2}-\frac{i \xi}{\lambda^{2}+\xi^{2} /\left(1+\xi^{2}\right)} \widehat{v}_{1} .
\end{aligned}
$$

By (15), we have

$$
\begin{aligned}
\left|(\lambda-\omega) \widehat{u}_{1}\right| & \leq\left|\frac{\lambda(\lambda-\omega)}{\lambda^{2}+\xi^{2} /\left(\xi^{2}+1\right)} \widehat{v}_{1}\right|+\left|\frac{(\lambda-\omega) \xi}{\lambda^{2}\left(\xi^{2}+1\right)+\xi^{2}} \widehat{v}_{2}\right| \\
& \leq\left|\widehat{v}_{1}\right|+\left|\frac{(\lambda-\omega) \xi}{\lambda^{2}\left(\xi^{2}+1\right)+\xi^{2}} \widehat{v}_{2}\right|
\end{aligned}
$$

Since $(\lambda-\omega) \xi /\left(\lambda^{2}\left(\xi^{2}+1\right)+\xi^{2}\right) \rightarrow 0$ as $|\xi| \rightarrow \infty$, there exists positive real number $N_{1}$, when $|\xi| \geq N_{1}$, such that $\mid(\lambda-$ $\omega) \xi /\left(\lambda^{2}\left(\xi^{2}+1\right)+\xi^{2}\right) \mid \leq 1$; that is, $\left|(\lambda-\omega) \widehat{u}_{1}\right| \leq\left|\widehat{v}_{1}\right|+\left|\widehat{v}_{2}\right|$.

Solving the inequality $\left|(\lambda-\omega) \xi /\left(\lambda^{2}\left(\xi^{2}+1\right)+\xi^{2}\right)\right| \leq \mid(\lambda-$ $\omega) \xi / \lambda^{2} \mid \leq 1$ when $|\xi| \leq N_{1}$, we can obtain that when $\omega \geq$ $N_{1} / 4$,

$$
\left|(\lambda-\omega) \widehat{u}_{1}\right| \leq\left|\widehat{v}_{1}\right|+\left|\widehat{v}_{2}\right|
$$


By (16), we know

$$
\begin{aligned}
\left|(\lambda-\omega) \widehat{u}_{2}\right| \leq & \left|\frac{\lambda(\lambda-\omega)}{\lambda^{2}+\xi^{2} /\left(\xi^{2}+1\right)} \widehat{v}_{2}\right| \\
& +\left|\frac{(\lambda-\omega) \xi}{\lambda^{2}+\xi^{2} /\left(\xi^{2}+1\right)} \widehat{v}_{1}\right| \\
& \leq\left|\widehat{v}_{2}\right|+\left|\frac{(\lambda-\omega) \xi}{\lambda^{2}} \widehat{v}_{1}\right|
\end{aligned}
$$

Since $(\lambda-\omega)|\xi| / \lambda^{2} \rightarrow 0$ as $|\xi| \rightarrow 0$, there exists a positive real number $N_{2}$, when $|\xi| \leq N_{2}$, such that $\left|(\lambda-\omega) \xi / \lambda^{2}\right| \leq 1$; that is, $\left|(\lambda-\omega) \widehat{u}_{2}\right| \leq\left|\widehat{v}_{2}\right|+\left|\widehat{v}_{1}\right|$.

Solving the inequality $(\lambda-\omega)|\xi| / \lambda^{2} \leq 1$ when $|\xi| \geq N_{2}$, we can obtain that when $\omega \geq N_{2} / 4$,

$$
\left|(\lambda-\omega) \widehat{u}_{2}\right| \leq\left|\widehat{v}_{2}\right|+\left|\widehat{v}_{1}\right| \text {. }
$$

Combining (18) and (20) and choosing $\omega=\operatorname{Max}\left\{N_{1} / 4\right.$, $\left.N_{2} / 4\right\}$, then we get (14) due to the definition of the operator norm.

In conclusion, we can obtain Lemma 4 from Lemmas 2 and 3.

\section{General Results for the Orbital Stability of Solitary Wave Solutions}

Equation (4) can be written in a Hamiltonian form

$$
\frac{d \vec{u}}{d t}=J E^{\prime}(\vec{u})
$$

where

$$
\begin{aligned}
& J=\frac{\partial}{\partial x}\left(\begin{array}{cc}
(1-\Delta)^{-1} & 0 \\
0 & 1
\end{array}\right), \quad \Delta=\frac{\partial^{2}}{\partial x^{2}} \\
& E(\vec{u})=-\int_{R}\left(v u+\frac{b_{2}}{3} u^{3}+\frac{b_{3}}{4} u^{4}\right) d x, \\
& E^{\prime}(\vec{u})=\left(\begin{array}{c}
E^{\prime}(u) \\
E^{\prime}(v)
\end{array}\right)=\left(\begin{array}{c}
-v-b_{2} u^{2}-b_{3} u^{3} \\
-u
\end{array}\right) .
\end{aligned}
$$

Let $X=H^{1}(R) \times L^{2}(R)$, whose dual space is $X^{*}=$ $H^{-1}(R) \times L^{2}(R)$, and the inner product of $X$ is

$$
\begin{array}{r}
\left(\vec{u}_{1}, \vec{u}_{2}\right)=\int_{R}\left(u_{1} u_{2}+v_{1} v_{2}+v_{1 x} v_{2 x}\right) d x, \\
\forall \vec{u}_{1}, \vec{u}_{2} \in X .
\end{array}
$$

There exists a natural isomorphism $I: X \rightarrow X^{*}$ defined by $\left\langle I \vec{u}_{1}, \vec{u}_{2}\right\rangle=\left(\vec{u}_{1}, \vec{u}_{2}\right)$, where $\langle\cdot, \cdot\rangle$ denotes the pairing between $X$ and $X^{*}$, and

$$
\left\langle\vec{u}_{1}, \vec{u}_{2}\right\rangle=\int_{R}\left(u_{1} u_{2}+v_{1} v_{2}\right) d x
$$

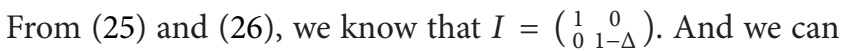
verify that $J$ is an skew symmetric operator; that is, $\langle J \vec{u}, \vec{v}\rangle=$ $-\langle\vec{u}, J \vec{v}\rangle$.
Let $T$ be a one-parameter group of unitary operator on $X$ defined by $T(s) \vec{u}(\cdot)=\vec{u}(\cdot-s)$, where $\vec{u}(s) \in X$, for all $s \in R$. Obviously, $T^{\prime}(0)=\left(\begin{array}{cc}-\partial / \partial x & 0 \\ 0 & -\partial / \partial x\end{array}\right)$. Since $T^{\prime}(0)=J B$, we can get $B=\left(\begin{array}{cc}\Delta-1 & 0 \\ 0 & -1\end{array}\right)$.

Therefore, we define

$$
\begin{aligned}
V(\vec{u}) & =-\frac{1}{2}\langle B \vec{u}, \vec{u}\rangle=-\frac{1}{2}\left\langle\left(\begin{array}{cc}
\Delta-1 & 0 \\
0 & -1
\end{array}\right)\left(\begin{array}{l}
u \\
v
\end{array}\right),\left(\begin{array}{l}
u \\
v
\end{array}\right)\right\rangle \\
& =\frac{1}{2}\left\langle\left(\begin{array}{c}
u-u_{x x} \\
v
\end{array}\right),\left(\begin{array}{l}
u \\
v
\end{array}\right)\right\rangle \\
& =\frac{1}{2} \int_{R}\left(u^{2}-u_{x x} u+v^{2}\right) d x .
\end{aligned}
$$

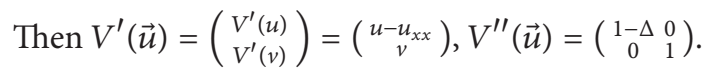

The solitary waves (6a) and (7a) of (4) can be written as

$$
\vec{\phi}_{i c}=\left(\begin{array}{l}
\varphi_{i c}(x-c t) \\
\psi_{i c}(x-c t)
\end{array}\right)=T(c t) \vec{\phi}_{i}(x), \quad i=1,2,
$$

where $\varphi_{1}(x)$ and $\varphi_{2}(x)$ are defined by (6b) and (7b), respectively. Now, we consider the orbital stability of solitary waves $T(c t) \vec{\phi}_{i}(x)$. Avoiding repetition, we let $\vec{\phi}_{c}(x)$ be one of $\vec{\phi}_{1}(x)$ and $\vec{\phi}_{2}(x)$. We will verify that (4) and the solitary wave $T(c t) \vec{\phi}_{c}(x)$ satisfy the three assumption conditions of the orbital stability theory presented by Grillakis et al. in [10].

Verification of Assumption 1. From Lemma 4 in Section 2, we obtain that the initial value problem of (4) has a unique solution. And it is easy to prove that $E(\vec{u})$ and $V(\vec{u})$ defined by (23) and (27) satisfy

$$
\begin{aligned}
& E(\vec{u}(t))=E(\vec{u}(0))=E\left(\vec{u}_{0}\right), \\
& V(\vec{u}(t))=V(\vec{u}(0))=V\left(\vec{u}_{0}\right),
\end{aligned}
$$

respectively.

This shows that (4) satisfies the Assumption 1 in [10].

Verification of Assumption 2. Firstly, we can prove the following lemma.

Lemma 5. $\vec{\phi}_{c}$ is a bounded state solution of (4), satisfying $E^{\prime}\left(\vec{\phi}_{c}\right)+c V^{\prime}\left(\vec{\phi}_{c}\right)=0$.

Proof. Substituting the solution $\vec{\phi}_{c}=\left(\begin{array}{l}\varphi_{c} \\ \psi_{c}\end{array}\right)$ into (4), we obtain

$$
\begin{gathered}
\varphi_{c \xi}=c \psi_{c \xi}, \\
-c \varphi_{c \xi \xi \xi}+c \varphi_{c \xi}=\left(\psi_{c}+b_{2} \varphi_{c}^{2}+b_{3} \varphi_{c}^{3}\right)_{\xi}
\end{gathered}
$$

Integrating (30) once, we get

$$
\begin{gathered}
\varphi_{c}=c \psi_{c}+a_{1}, \\
-c \varphi_{c \xi \xi}+c \varphi_{c}=\left(\psi_{c}+b_{2} \varphi_{c}^{2}+b_{3} \varphi_{c}^{3}\right)+a_{2},
\end{gathered}
$$

where $a_{1}, a_{2}$ are integral constants. 


$$
\begin{gathered}
\varphi_{c}=c \psi_{c}, \\
-c \varphi_{c \xi \xi}+c \varphi_{c}=\psi_{c}+b_{2} \varphi_{c}^{2}+b_{3} \varphi_{c}^{3} .
\end{gathered}
$$$$
\varphi_{c}, \psi_{c}, \varphi_{c \xi \xi} \rightarrow 0 \text { as }|\xi| \rightarrow \infty \text {, so } a_{1}=0 \text { and } a_{2}=0 \text {. Thus }
$$

Furthermore,

$$
\begin{aligned}
E^{\prime}\left(\vec{\phi}_{c}\right) & +c V^{\prime}\left(\vec{\phi}_{c}\right) \\
= & \left(\begin{array}{c}
-\psi_{c}-b_{2} \varphi_{c}^{2}-b_{3} \varphi_{c}^{3} \\
-\varphi_{c}
\end{array}\right)+c\left(\begin{array}{c}
\varphi_{c}-\varphi_{c \xi \xi} \\
\psi_{c}
\end{array}\right) \\
= & \left(\begin{array}{c}
-\psi_{c}-b_{2} \varphi_{c}^{2}-b_{3} \varphi_{c}^{3}-c \varphi_{c \xi \xi}+c \varphi_{c} \\
-\varphi_{c}+c \psi_{c}
\end{array}\right) .
\end{aligned}
$$

Due to (32), we have $E^{\prime}\left(\vec{\phi}_{c}\right)+c V^{\prime}\left(\vec{\phi}_{c}\right)=0$.

The above Lemma 5 shows that (4) has the bounded state solutions, and the two solitary waves $\vec{\phi}_{1 c}$ and $\vec{\phi}_{2 c}$ given in Theorem 1 both are the bounded state solutions of the equation.

Verification of Assumption 3. We consider spectrum analysis of the operator $H_{c}$.

Now we define the operator $H_{c}: X \rightarrow X^{*}$ as $H_{c}=$ $E^{\prime \prime}\left(\vec{\phi}_{c}\right)+c V^{\prime \prime}\left(\vec{\phi}_{c}\right)$, where

$$
E^{\prime \prime}(\vec{u})=\left(\begin{array}{cc}
-2 b_{2} u-3 b_{3} u^{2} & -1 \\
-1 & 0
\end{array}\right), \quad V^{\prime \prime}(\vec{u})=\left(\begin{array}{cc}
1-\Delta & 0 \\
0 & 1
\end{array}\right) .
$$

Therefore,

$$
\begin{aligned}
H_{c} & =E^{\prime \prime}\left(\vec{\phi}_{c}\right)+c V^{\prime \prime}\left(\vec{\phi}_{c}\right) \\
& =\left(\begin{array}{cc}
-2 b_{2} \varphi_{c}-3 b_{3} \varphi_{c}^{2} & -1 \\
-1 & 0
\end{array}\right)+c\left(\begin{array}{cc}
1-\Delta & 0 \\
0 & 1
\end{array}\right) \\
& =\left(\begin{array}{rr}
-2 b_{2} \varphi_{c}-3 b_{3} \varphi_{c}^{2}-c \frac{\partial^{2}}{\partial x^{2}}+c & -1 \\
-1 & c
\end{array}\right) .
\end{aligned}
$$

For any $\vec{u}_{1}, \vec{u}_{2} \in H^{1}(R) \times L^{2}(R)$, we have $\left\langle H_{c} \vec{u}_{1}, \vec{u}_{2}\right\rangle=$ $\left\langle H_{c} \vec{u}_{2}, \vec{u}_{1}\right\rangle$. This means that $H_{c}$ is a self-conjugate operator, that is, $H_{c}=H_{c}^{*}$, and that $I^{-1} H_{c}$ is a bounded self-conjugate operator on $X$. The eigenvalues of $H_{c}$ consist of the real numbers $\lambda$ which ensure that $H_{c}-\lambda I$ are irreversible.

From (30), we have $-c \varphi_{c \xi \xi \xi}+c \varphi_{c \xi}=\left((1 / c) \varphi_{c}+b_{2} \varphi_{c}^{2}+\right.$ $\left.b_{3} \varphi_{c}^{3}\right)_{\xi}$. Namely,

$$
\left[-2 b_{2} \varphi_{c}-3 b_{3} \varphi_{c}^{2}-c \frac{\partial^{2}}{\partial x^{2}}+\left(c-\frac{1}{c}\right)\right] \varphi_{c x}=0
$$

Let $L=-2 b_{2} \varphi_{c}-3 b_{3} \varphi_{c}^{2}-c\left(\partial^{2} / \partial x^{2}\right)+(c-(1 / c))$. Since the existence of solitary wave solution $\vec{\phi}_{c}=\left(\begin{array}{l}\varphi_{c} \\ \psi_{c}\end{array}\right)$ of (4) is based on the condition that $c^{2}-1>0, c-1 / c>0$ as $c>1$, and $-2 b_{2} \varphi_{c}-3 b_{3} \varphi_{c}^{2} \rightarrow 0$ as $|\xi| \rightarrow \infty$, it is easy to know that $\sigma_{\text {ess }}(L)=[c-1 / c,+\infty)$ by Weyl's essential spectrum theorem.
Moreover, from (36), we have $L \varphi_{c x}=0$, where $x=0$ is a unique zero point of $\varphi_{c x}$. By Sturm-Liouville theorem we know that zero is the second eigenvalue of $L$. Thus $L$ only has one strictly negative eigenvalue $-\sigma^{2}$ in the case of $c>1$, whose corresponding eigenfunction is denoted by $\chi$; that is, $L \chi=-\sigma^{2} \chi$.

Therefore, $H_{c}$ has a unique simple negative eigenvalue, and zero is its eigenvalue and the rest of its spectrums are bounded away from zero. So, $H_{c}$ satisfies the Assumption 3 in $[10]$.

According to $[10,11]$, we can get the following lemma.

Lemma 6. For any real function $y \in H^{1}(R)$ with $\langle y, \chi\rangle=$ $\left\langle y, \varphi_{c x}\right\rangle=0$, there exists $\delta>0$ such that $\langle L y, y\rangle \geq \delta\|y\|_{H^{2}(R)}^{2}$. Let $\vec{\chi}_{c}=\left(\begin{array}{c}\chi \\ (1 / c) \chi\end{array}\right)$. We have

$$
\begin{aligned}
& \left\langle H_{c} \vec{\chi}_{c}, \vec{\chi}_{c}\right\rangle \\
& \quad=\int_{R}\left[-2 b_{2} \varphi_{c} \chi^{2}-3 b_{3} \varphi_{c}^{2} \chi^{2}-c \chi_{x x} \chi+\left(c-\frac{1}{c}\right) \chi^{2}\right] d x \\
& \quad=\langle L \chi, \chi\rangle \\
& \quad=-\sigma^{2}\langle\chi, \chi\rangle<0 .
\end{aligned}
$$

Let $\vec{\varphi}_{c x}=\left(\begin{array}{c}\varphi_{c x} \\ (1 / c) \varphi_{c x}\end{array}\right)$, and then

$$
\begin{aligned}
& \left\langle H_{c} \vec{\varphi}_{c x}, \vec{\varphi}_{c x}\right\rangle \\
& =\int_{R}\left[-2 b_{2} \varphi_{c} \varphi_{c x}^{2}-3 b_{3} \varphi_{c}^{2} \varphi_{c x}^{2}-c \varphi_{c x x x} \varphi_{c x}+\left(c-\frac{1}{c}\right) \varphi_{c x}^{2}\right] d x \\
& =\left\langle L \varphi_{c x}, \varphi_{c x}\right\rangle=0 .
\end{aligned}
$$

Le

$$
\begin{gathered}
P=\left\{\vec{p}_{c} \in X \mid \vec{p}_{c}=\left(\begin{array}{c}
p_{1} \\
p_{2}
\end{array}\right),\left\langle p_{1}, \chi\right\rangle=\left\langle p_{1}, \varphi_{c x}\right\rangle=0,\right. \\
\left.\left\langle p_{2}, \frac{1}{c} \chi\right\rangle=\left\langle p_{2}, \frac{1}{c} \varphi_{c x}\right\rangle=0\right\} .
\end{gathered}
$$

We have

$$
\begin{aligned}
& \left\langle H_{c} \vec{p}_{c}, \vec{p}_{c}\right\rangle \\
& =\int_{R}\left[-2 b_{2} \varphi_{c} p_{1}^{2}-3 b_{3} \varphi_{c}^{2} p_{1}^{2}\right. \\
& \left.\quad-c p_{1 x x} p_{1}+\left(c-\frac{1}{c}\right) p_{1}^{2}+\frac{1}{c}\left(p_{1}-c p_{2}\right)^{2}\right] d x .
\end{aligned}
$$

Thus $\left\langle H_{c} \vec{p}_{c}, \vec{p}_{c}\right\rangle \geq\left\langle L p_{1}, p_{1}\right\rangle \geq \delta\left\|\vec{p}_{c}\right\|^{2}>0$ for any $\vec{p}_{c} \in P$ when $c>1$. 
According to the above analysis, when $c>1$, we can make spectrum decomposition for $\mathrm{H}_{c}$. Let

$$
\begin{gathered}
N=\left\{k_{1} \vec{\chi}_{c} \mid k_{1} \in R\right\}, \\
Z=\left\{k_{2} \vec{\varphi}_{c x} \mid k_{2} \in R\right\}, \\
P=\left\{\vec{p}_{c} \in X \mid \vec{p}_{c}=\left(\begin{array}{c}
p_{1} \\
p_{2}
\end{array}\right),\left\langle p_{1}, \chi\right\rangle=\left\langle p_{1}, \varphi_{c x}\right\rangle=0,\right. \\
\left.\left\langle p_{2}, \frac{1}{c} \chi\right\rangle=\left\langle p_{2}, \frac{1}{c} \varphi_{c x}\right\rangle=0\right\} .
\end{gathered}
$$

For any $\vec{u} \in N, \vec{u} \neq 0,\left\langle H_{c} \vec{u}, \vec{u}\right\rangle<0$ due to $\left\langle H_{c} k_{1} \vec{\chi}_{c}\right.$, $\left.k_{1} \vec{\chi}_{c}\right\rangle=k_{1}^{2}\left\langle H_{c} \vec{\chi}_{c}, \vec{\chi}_{c}\right\rangle=-k_{1}^{2} \sigma^{2}\langle\chi, \chi\rangle<0$.

For any $\vec{z} \in Z, \vec{z} \neq 0,\left\langle H_{c} \vec{z}, \vec{z}\right\rangle=0$ due to $\left\langle H_{c} k_{2} \vec{\varphi}_{c x}\right.$, $\left.k_{2} \vec{\varphi}_{c x}\right\rangle=0$.

For any $\vec{p}_{c} \in P, \vec{p}_{c} \neq 0,\left\langle H_{c} \vec{p}_{c}, \vec{p}_{c}\right\rangle>\delta\left\|\vec{p}_{c}\right\|_{H^{2}(R)}^{2}$.

Thus the space $X$ can be decomposed as a direct sum $X=$ $N+Z+P$, where $Z$ is the kernel space of $H_{c}, N$ is a finitedimensional subspace and $P$ is a closed subspace.

We now define $d(c): R \rightarrow R$ as $d(c)=E\left(\vec{\phi}_{c}\right)+c V\left(\vec{\phi}_{c}\right)$, and then

$$
d^{\prime}(c)=\left\langle E^{\prime}\left(\vec{\phi}_{c}\right), \vec{\phi}_{c}^{\prime}\right\rangle+c\left\langle V^{\prime}\left(\vec{\phi}_{c}\right), \vec{\phi}_{c}^{\prime}\right\rangle+V\left(\vec{\phi}_{c}\right)=V\left(\vec{\phi}_{c}\right) .
$$

According to the above verification of Assumptions 1-3, (4) and its solitary wave solutions satisfy the three assumptions of Theorem 2 in [10], so we can obtain the following general conclusion on orbital stability of solitary waves for (4).

Theorem 7. Suppose that $b_{2} b_{3} \neq 0, b_{3} \geq 0, c>1$, and $\vec{\phi}_{c}=$ $T(c t) \vec{\phi}(x)$ is the solitary wave solution of (4). Then,

(1) $\vec{\phi}_{c}$ is orbitally stable as $d^{\prime \prime}(c)>0$;

(2) $\vec{\phi}_{c}$ is orbitally unstable as $d^{\prime \prime}(c)<0$.

Remark 8. The proof of the conclusion (2) in Theorem 7 will be given by Theorem 26 in Section 5 .

\section{Orbital Stability and \\ Influence of the Interaction between Nonlinear Terms on It}

In this section, by using two exact solitary waves (6a), (6b), and $(6 c)$ and $(7 a),(7 b)$, and $(7 c)$ given in Theorem 1 , we will give the explicit expressions for the discrimination $d_{i}^{\prime \prime}(c)$. Then with the analysis method, we will give several sufficient conditions to judge the orbital stability and instability of the solitary waves. Furthermore, we will also analyze the influence of the interaction between two nonlinear terms on the orbital stability. We assume that $b_{3}>0$ and $c>1$ in this section.
4.1. Discrimination $d_{i}^{\prime \prime}(c)$. In view of (42), we have

$$
\begin{aligned}
d_{i}^{\prime}(c) & =V\left(\vec{\phi}_{i c}\right) \\
& =\frac{1}{2} \int_{R}\left(\varphi_{i c}^{2}-\varphi_{i c x x} \varphi_{i c}+\psi_{i c}^{2}\right) d x, \quad i=1,2 .
\end{aligned}
$$

Next, we simplify (43). According to (6a) and (7a) in Theorem 1, we have $\psi_{i}=(1 / c) \varphi_{i}$. Substituting it into (43), and letting $z=e^{\alpha_{i} \xi}\left(\alpha_{i}>0\right)$, we obtain

$$
\begin{aligned}
& d_{i}^{\prime}(c)=\frac{1}{2} \int_{R}\left(\varphi_{i c}^{2}-\varphi_{i c x x} \varphi_{i c}+\psi_{i c}^{2}\right) d x \\
& =\frac{1}{2} \int_{R}\left[\left(1+\frac{1}{c^{2}}\right) \varphi_{i c}^{2}+\varphi_{i c x}^{2}\right] d x \\
& =\frac{1}{2} \int_{R}\left[\left(1+\frac{1}{c^{2}}\right) \varphi_{i c}^{2}+\varphi_{i c \xi}^{2}\right] d \xi \\
& =\frac{1}{2 \alpha_{i}} \int_{0}^{+\infty} \frac{1}{z}\left[\frac{2 A_{i} z}{(1+z)^{2}+2 B_{i} z}\right]^{2} \\
& \times\left\{1+\frac{1}{c^{2}}+\left[\frac{\alpha_{i}\left(z^{2}-1\right)}{(1+z)^{2}+2 B_{i} z}\right]^{2}\right\} d z \\
& =\left(1+\frac{1}{c^{2}}\right) \frac{2 A_{i}^{2}}{\alpha_{i}} \int_{0}^{+\infty} \frac{z}{\left[(1+z)^{2}+2 B_{i} z\right]^{2}} d z \\
& +2 A_{i}^{2} \alpha_{i} \int_{0}^{+\infty} \frac{z\left(z^{2}-1\right)^{2}}{\left[(1+z)^{2}+2 B_{i} z\right]^{4}} d z,
\end{aligned}
$$

where $\alpha_{i}, A_{i}, B_{i}$ are given by (6c) and (7c).

Since $-2<B_{i}<0$, we can solve above two integrations. Then,

$$
\begin{aligned}
& d_{i}^{\prime}(c) \\
& =\left(1+\frac{1}{c^{2}}\right) \\
& \times A_{i}^{2}\left\{\frac{B_{i}+1}{\sqrt{-B_{i}\left(B_{i}+2\right)}}\right. \\
& \left.\times\left[\pi-2 \arctan \left(\frac{B_{i}+1}{\sqrt{-B_{i}\left(B_{i}+2\right)}}\right)\right]-2\right\} \\
& \times\left(2 \alpha_{i} B_{i}\left(B_{i}+2\right)\right)^{-1}
\end{aligned}
$$




$$
\begin{array}{rlrl}
+A_{i}^{2} \alpha_{i}\left\{2\left(3+2 B_{i}+B_{i}^{2}\right)-3 \frac{B_{i}+1}{\sqrt{-B_{i}\left(B_{i}+2\right)}}\right. & =\frac{d_{2}^{\prime \prime}(c)}{k\left(3 c-3 / c-k^{2}\right) D_{2}} \\
& \left.\times\left[\pi-2 \arctan \left(\frac{B_{i}+1}{\sqrt{-B_{i}\left(B_{i}+2\right)}}\right)\right]\right\} & +\frac{\sqrt{c^{2}-1}\left(16 c^{4}+7 k^{2} c^{3}-\left(8+3 k^{4}\right) c^{2}-7 k^{2} c+16\right)}{6 b_{3} c^{3}\left(c^{2}-1+k^{2} c\right)},
\end{array}
$$$$
\times\left(12 B_{i}^{2}\left(B_{i}+2\right)^{2}\right)^{-1} .
$$

If

$$
B_{i}=B_{1}=-1+\frac{\sqrt{2} b_{2} c}{\sqrt{c\left[2 b_{2}^{2} c+9 b_{3}\left(c^{2}-1\right)\right]}},
$$

then (45) can be simplified into the following form:

$$
\begin{aligned}
& d_{1}^{\prime}(c) \\
& =\frac{1}{3 \sqrt{b_{3} c}}\left\{6 \alpha \sqrt{b_{3} c}-\sqrt{2} b_{2}\right. \\
& \quad \times\left[\pi-2 \arctan \left(\frac{\sqrt{2} b_{2}}{3} \sqrt{\left.\left.\left.\frac{c}{b_{3}\left(c^{2}-1\right)}\right)\right]\right\}}\right.\right. \\
& \cdot\left(\frac{1}{2 b_{3} c}+\frac{3 c}{2 b_{3}}+\frac{b_{2}^{2}}{9 b_{3}^{2}}\right)-\frac{c \alpha^{3}}{3 b_{3}} .
\end{aligned}
$$

If

$$
B_{i}=B_{2}=-1-\frac{\sqrt{2} b_{2} c}{\sqrt{c\left[2 b_{2}^{2} c+9 b_{3}\left(c^{2}-1\right)\right]}},
$$

then (45) can be simplified into the following form:

$$
\begin{aligned}
& d_{2}^{\prime}(c) \\
& =\frac{1}{3 \sqrt{b_{3} c}}\left\{6 \alpha \sqrt{b_{3} c}+\sqrt{2} b_{2}\right. \\
& \left.\quad \times\left[\pi+2 \arctan \left(\frac{\sqrt{2} b_{2}}{3} \sqrt{\frac{c}{b_{3}\left(c^{2}-1\right)}}\right)\right]\right\} \\
& \quad\left(\frac{1}{2 b_{3} c}+\frac{3 c}{2 b_{3}}+\frac{b_{2}^{2}}{9 b_{3}^{2}}\right)-\frac{c \alpha^{3}}{3 b_{3}} .
\end{aligned}
$$

By calculating, we have

$$
\begin{aligned}
& d_{1}^{\prime \prime}(c) \\
& =-\frac{k\left(3 c-3 / c-k^{2}\right) D_{1}}{4 b_{3} c^{3 / 2}} \\
& \quad+\frac{\sqrt{c^{2}-1}\left(16 c^{4}+7 k^{2} c^{3}-\left(8+3 k^{4}\right) c^{2}-7 k^{2} c+16\right)}{6 b_{3} c^{3}\left(c^{2}-1+k^{2} c\right)},
\end{aligned}
$$

where

$$
\begin{gathered}
k=\frac{\sqrt{2} b_{2}}{3 \sqrt{b_{3}}}, \quad D_{1}=\pi-2 \arctan \left(k \sqrt{\frac{c}{c^{2}-1}}\right), \\
D_{2}=\pi+2 \arctan \left(k \sqrt{\frac{c}{c^{2}-1}}\right) .
\end{gathered}
$$

Furthermore, suppose that

$$
\begin{aligned}
M_{1} & =k \sqrt{\frac{c}{c^{2}-1}} D_{1} \\
& =k \sqrt{\frac{c}{c^{2}-1}}\left[\pi-2 \arctan \left(k \sqrt{\frac{c}{c^{2}-1}}\right)\right], \\
M_{2} & =k \sqrt{\frac{c}{c^{2}-1}} D_{2} \\
& =k \sqrt{\frac{c}{c^{2}-1}}\left[\pi+2 \arctan \left(k \sqrt{\frac{c}{c^{2}-1}}\right)\right] .
\end{aligned}
$$

Then (50) can be written as

$$
\begin{aligned}
& d_{1}^{\prime \prime}(c) \\
& =\frac{\sqrt{c^{2}-1}}{12 b_{3} c^{2}} \\
& \quad \times\left[-3 M_{1}\left(3 c-\frac{3}{c}-k^{2}\right)\right. \\
& \left.\quad+\frac{2\left(16 c^{4}+7 k^{2} c^{3}-\left(8+3 k^{4}\right) c^{2}-7 k^{2} c+16\right)}{c\left(c^{2}-1+k^{2} c\right)}\right] .
\end{aligned}
$$

And (51) can be written as

$$
\begin{aligned}
& d_{2}^{\prime \prime}(c) \\
& =\frac{\sqrt{c^{2}-1}}{12 b_{3} c^{2}} \\
& \times\left[3 M_{2}\left(3 c-\frac{3}{c}-k^{2}\right)\right. \\
& \left.\quad+\frac{2\left(16 c^{4}+7 k^{2} c^{3}-\left(8+3 k^{4}\right) c^{2}-7 k^{2} c+16\right)}{c\left(c^{2}-1+k^{2} c\right)}\right] .
\end{aligned}
$$


Therefore, we only need to consider the conditions such that $d_{i}^{\prime \prime}(c)>0$ hold in (54) and (55) to study the orbital stability of the solitary waves $\vec{\phi}_{i c}$, while needing to consider $d_{i}^{\prime \prime}(c)<0$ to study instability.

4.2. Discussion on $M_{1}$ and $M_{2}$. In this section, we consider $M_{1}$ and $M_{2}$ in the case of $c>1$ and $b_{3}>0$.

(1) For $M_{1}$. If $b_{2}>0$, then $k>0$. Suppose that $x=$ $k \sqrt{c /\left(c^{2}-1\right)} \in(0,+\infty)$, and then

$$
\begin{aligned}
& \lim _{c \rightarrow+\infty} x=\lim _{c \rightarrow+\infty} k \sqrt{\frac{c}{c^{2}-1}}=0, \\
& \lim _{c \rightarrow 1} x=\lim _{c \rightarrow 1} k \sqrt{\frac{c}{c^{2}-1}}=+\infty .
\end{aligned}
$$

Let $g(x)=x(\pi-2 \arctan x)$. We have

$$
\begin{gathered}
g^{\prime}(x)=\pi-2 \arctan x-\frac{2 x}{1+x^{2}}, \\
g^{\prime \prime}(x)=-\frac{4}{\left(1+x^{2}\right)^{2}}<0 .
\end{gathered}
$$

Moreover, $g(x)$ can obtain the local maximum at $x_{0}$, where $x_{0}$ satisfies $g^{\prime}\left(x_{0}\right)=0$ and

$$
g\left(x_{0}\right)=x_{0}\left(\pi-2 \arctan x_{0}\right)=\frac{2 x_{0}^{2}}{1+x_{0}^{2}}<2 .
$$

Since

$$
\begin{array}{r}
\lim _{x \rightarrow+\infty} M_{1}=\lim _{x \rightarrow+\infty} g(x)=\lim _{x \rightarrow+\infty} x(\pi-2 \arctan x)=2, \\
M_{1} \in(0,2) .
\end{array}
$$

When $b_{2}<0$, it is easy to know that $x \in(-\infty, 0)$, and then $M_{1} \in(-\infty, 0)$.

(2) For $M_{2}$. When $b_{2}>0$, it is clear that $x \in(0,+\infty)$, and then $M_{2} \in(0,+\infty)$. But if $b_{2}<0$, then $k<0$ and $x=k \sqrt{c /\left(c^{2}-1\right)} \in(-\infty, 0)$. Therefore

$$
\lim _{x \rightarrow-\infty} M_{2}=\lim _{x \rightarrow-\infty} x(\pi+2 \arctan x)=-2 .
$$

Similarly, we can get $M_{2} \in(-2,0)$.

4.3. Orbital Stability of Solitary Waves for (4) in the Case of $3 c-3 / c-k^{2}>0$. Based on (54), (55), and above discussion on $M_{1}, M_{2}$, we want to obtain much more simple conditions on the orbital stability of solitary waves $\vec{\phi}_{1 c}$ and $\vec{\phi}_{2 c}$.

4.3.1. Orbital Stability of $\vec{\phi}_{1 c}$. (1) If $b_{2}>0$, then $M_{1} \in(0,2)$. At this time, $-3 M_{1}\left(3 c-3 / c-k^{2}\right)<0$. In order to find $c$ such that $d_{1}^{\prime \prime}(c)>0$, we only need to consider $M_{1}=2$ in (54). It is easy to see that $d_{1}^{\prime \prime}(c)>0$ when $c$ satisfies

$$
7 c^{4}+k^{2} c^{3}+10 c^{2}-k^{2} c+7>0 .
$$

Thus, $\vec{\phi}_{1 c}$ is orbitally stable.
If $b_{2}<0,-3 M_{1}\left(3 c-3 / c-k^{2}\right)>0$. In order to make $d_{1}^{\prime \prime}(c)>0$, that is, $\vec{\phi}_{1 c}$ is orbitally stable, only when $c$ satisfies

$$
16 c^{4}+7 k^{2} c^{3}-\left(8+3 k^{4}\right) c^{2}-7 k^{2} c+16>0 .
$$

(2) If $b_{2}>0$, then $M_{1} \in(0,2)$. Here, $-3 M_{1}\left(3 c-3 / c-k^{2}\right)<$ 0 in (54). In order to make $d_{1}^{\prime \prime}(c)<0$, we only need to consider $M_{1}=0$ in (54). Then, it is easy to see that $d_{1}^{\prime \prime}(c)<0$ when $c$ satisfies

$$
16 c^{4}+7 k^{2} c^{3}-\left(8+3 k^{4}\right) c^{2}-7 k^{2} c+16<0 .
$$

Thus, $\vec{\phi}_{1 c}$ is orbitally unstable.

4.3.2. Orbital Stability of $\vec{\phi}_{2 c}$. (1) If $b_{2}>0$, then $M_{2} \epsilon$ $(0,+\infty)$. In order to make $d_{2}^{\prime \prime}(c)>0$, it is easy to know that $\vec{\phi}_{2 c}$ is orbitally stable if $c$ satisfies (62).

If $b_{2}<0$, then $M_{2} \in(-2,0)$. In order to make $d_{2}^{\prime \prime}(c)>0$, we only need to consider $M_{2}=-2$ in (55). It is easy to know that $\vec{\phi}_{2 c}$ is orbitally stable if $c$ satisfies (61).

(2) If $b_{2}<0$, in order to make $d_{2}^{\prime \prime}(c)<0$, we only need to consider $M_{2}=0$ in (55). Then, it is easy to know that $\vec{\phi}_{2 c}$ is orbitally unstable if $c$ satisfies (63).

In addition, we know that $3 c-3 / c-k^{2}>0$ is equal to $c>$ $\left(1 / 27 b_{3}\right)\left[b_{2}^{2}+\sqrt{b_{2}^{4}+729 b_{3}^{2}}\right]$ or $c<\left(1 / 27 b_{3}\right)\left[b_{2}^{2}-\sqrt{b_{2}^{4}+729 b_{3}^{2}}\right]$, but we always assume $c>1$ through this section. So if we assume $c_{0}=\left(1 / 27 b_{3}\right)\left[b_{2}^{2}+\sqrt{b_{2}^{4}+729 b_{3}^{2}}\right]$, then $3 c-3 / c-k^{2}>0$ is equal to $c>c_{0}$.

Summarizing above results, we have the following theorem.

Theorem 9. Suppose that $b_{3}>0$ and $c>c_{0}$, where $c_{0}=\left(b_{2}^{2}+\right.$ $\left.\sqrt{b_{2}^{4}+729 b_{3}^{2}}\right) / 27 b_{3}>1$.

(1) $\vec{\phi}_{1 c}$ is orbitally stable if $b_{2}>0$ and the wave speed $c$ satisfies (61), or $b_{2}<0$ and the wave speed $c$ satisfies (62); $\vec{\phi}_{1 c}$ is orbitally unstable if $b_{2}>0$ and the wave speed c satisfies (63).

(2) $\vec{\phi}_{2 c}$ is orbitally stable if $b_{2}>0$ and the wave speed $c$ satisfies (62), or $b_{2}<0$ and the wave speed $c$ satisfies (61); $\vec{\phi}_{2 c}$ is orbitally unstable if $b_{2}<0$ and the wave speed c satisfies (63).

\subsection{Orbital Stability of Solitary Waves for}

(4) in the Case of $3 c-3 / c-k^{2}<0$

4.4.1. Orbital Stability of $\vec{\phi}_{1 c}$. (1) If $b_{2}>0,-3 M_{1}(3 c-3 / c-$ $\left.k^{2}\right)>0$. In order to make $d_{1}^{\prime \prime}(c)>0$, we only need to consider $M_{1}=0$ in (54). It is easy to see that $\vec{\phi}_{1 c}$ is orbitally stable if c satisfies (62).

(2) If $b_{2}>0$. In order to find $c$ such that $d_{1}^{\prime \prime}(c)<0$, we only need to consider $M_{1}=2$ in (54). It is easy to see that $d_{1}^{\prime \prime}(c)<0$ when $c$ satisfies

$$
7 c^{4}+k^{2} c^{3}+10 c^{2}-k^{2} c+7<0 .
$$

Thus, $\vec{\phi}_{1 c}$ is orbitally unstable. 
If $b_{2}<0,-3 M_{1}\left(3 c-3 / c-k^{2}\right)<0$. In order to make $d_{1}^{\prime \prime}(c)<0$, it is easy to know that $\vec{\phi}_{1 c}$ is orbitally unstable if $c$ satisfies (63).

4.4.2. Orbital Stability of $\vec{\phi}_{2 c}$. (1) If $b_{2}<0$, then $M_{2} \in(-2,0)$. At this time, $3 M_{2}\left(3 c-3 / c-k^{2}\right)>0$. In order to find $c$ such that $d_{2}^{\prime \prime}(c)>0$, we only need to consider $M_{2}=0$ in (55). It is easy to see that $\vec{\phi}_{2 c}$ is orbitally stable if $c$ satisfies (62).

(2) If $b_{2}>0$, then $M_{2} \in(0,+\infty)$. In order to make $d_{2}^{\prime \prime}(c)<$ 0 , it is easy to know that $\vec{\phi}_{2 c}$ is orbitally unstable if $\mathrm{c}$ satisfies (63).

If $b_{2}<0$, then $M_{2} \in(-2,0)$. Here, $3 M_{2}\left(3 c-3 / c-k^{2}\right)>0$ in (55). In order to find $c$ such that $d_{2}^{\prime \prime}(c)<0$, we only need to consider $M_{2}=-2$ in (55). It is easy to see that $\vec{\phi}_{2 c}$ is orbitally unstable if $c$ satisfies (64).

Summarizing above results, we have the following theorem.

Theorem 10. Suppose that $b_{3}>0$ and $1<c<c_{0}$, where $c_{0}=\left(b_{2}^{2}+\sqrt{b_{2}^{4}+729 b_{3}^{2}}\right) / 27 b_{3}>1$.

(1) $\vec{\phi}_{1 c}$ is orbitally stable if $b_{2}>0$ and the wave speed $c$ satisfies (62). $\vec{\phi}_{1 c}$ is orbitally unstable if $b_{2}>0$ and the wave speed c satisfies (64), or $b_{2}<0$ and the wave speed c satisfies (63).

(2) $\vec{\phi}_{2 c}$ is orbitally stable if $b_{2}<0$ and the wave speed $c$ satisfies (62). $\vec{\phi}_{2 c}$ is orbitally unstable if $b_{2}>0$ and the wave speed c satisfies (63), or $b_{2}<0$ and the wave speed $c$ satisfies (64).

4.5. Corollaries and Influences of Nonlinear Terms on Orbital Stability of the Solitary Waves for (4). In this part, we will firstly consider the orbital stability of the solitary waves for (4) with only one nonlinear term. Secondly, we will discuss the effect of nonlinear terms on orbital stability of the solitary waves for (4).

Corollary 11. Suppose that $b_{3}>0$ and $c>1$. If $b_{2}=0$, (4) has the solitary wave solutions $\vec{\phi}_{i c}=\left(\begin{array}{c}\varphi_{i}(\xi) \\ \psi_{i}(\xi)\end{array}\right), i=1,2$, where

$$
\begin{aligned}
& \varphi_{1}(\xi)=\frac{A_{1} \operatorname{sech}^{2}\left(\alpha_{1} / 2\right) \xi}{2+B_{1} \operatorname{sech}^{2}\left(\alpha_{1} / 2\right) \xi}, \quad \psi_{1}(\xi)=\frac{1}{c} \varphi_{1}(\xi), \\
& \alpha_{1}=\frac{\sqrt{c^{2}-1}}{c}, \quad A_{1}=\frac{\sqrt{2\left(c^{2}-1\right)}}{\sqrt{b_{3} c}}, \quad B_{1}=-1, \\
& \varphi_{2}(\xi)=\frac{A_{2} \operatorname{sech}^{2}\left(\alpha_{2} / 2\right) \xi}{2+B_{2} \operatorname{sech}^{2}\left(\alpha_{2} / 2\right) \xi}, \quad \psi_{2}(\xi)=\frac{1}{c} \varphi_{2}(\xi), \\
& \alpha_{2}=\frac{\sqrt{c^{2}-1}}{c}, \quad A_{2}=\frac{\sqrt{2\left(c^{2}-1\right)}}{\sqrt{b_{3} c}}, \quad B_{2}=-1 .
\end{aligned}
$$

Under the given conditions, we can easily conclude that the solitary waves $\vec{\phi}_{i c}, i=1,2$, are both orbitally stable.
Proof. When $b_{2}=0$, the above solitary waves (65) of (4) can be deduced from Theorem 1 directly.

Actually, it is clear that $k=0$ as $b_{2}=0$. Substituting $k=0$ into (50) and (51), we have

$$
d_{1}^{\prime \prime}(c)=d_{2}^{\prime \prime}(c)=\frac{4}{3 b_{3} c^{3}} \frac{2 c^{4}-c^{2}+2}{\sqrt{c^{2}-1}} .
$$

We know that $2 c^{4}-c^{2}+2>0$ in $(66)$, so $d_{i}^{\prime \prime}(c)>0, i=$ 1,2 , if $c>1$ and $b_{3}>0$. Thus, we know that the solitary waves $\vec{\phi}_{i c}, i=1,2$, of (4) are both orbitally stable according to Theorem 7 .

Corollary 12. When $c>1$ and $b_{3}=0$, then (4) has the solitary wave solution $\vec{\phi}_{c}=\left(\begin{array}{l}\varphi(\xi) \\ \psi(\xi)\end{array}\right)$, where

$$
\begin{gathered}
\varphi(\xi)=\frac{A \operatorname{sech}^{2}(\alpha / 2) \xi}{2+B \operatorname{sech}^{2}(\alpha / 2) \xi}, \quad \psi(\xi)=\frac{1}{c} \varphi(\xi), \\
\alpha=\frac{\sqrt{c^{2}-1}}{c}, \quad A=\frac{3\left(c^{2}-1\right)}{b_{2} c}, \quad B=0 .
\end{gathered}
$$

Under the given conditions, we know that the solitary wave $\vec{\phi}_{c}$ of (4) is orbitally stable.

Proof. When $b_{3}=0$, the above solitary wave (67) of (4) can be deduced from Theorem 1 directly.

Moreover, similar to deducing (50) and (51), by calculating, we can obtain

$$
\begin{aligned}
d^{\prime}(c)= & \frac{1}{2} \int_{R}\left(\varphi_{c}^{2}-\varphi_{c x x} \varphi_{c}+\psi_{c}^{2}\right) d x \\
= & \frac{1}{2} \int_{R}\left[\left(1+\frac{1}{c^{2}}\right) \varphi_{c}^{2}+\varphi_{c x}^{2}\right] d x \\
= & \frac{1}{2} \int_{R}\left[\left(1+\frac{1}{c^{2}}\right) \varphi_{c}^{2}+\varphi_{c \xi}^{2}\right] d \xi \\
= & \frac{1}{2 \alpha} \int_{0}^{+\infty} \frac{1}{z}\left[\frac{2 A z}{(1+z)^{2}+2 B z}\right]^{2} \\
& \times\left\{1+\frac{1}{c^{2}}+\left[\frac{\alpha\left(z^{2}-1\right)}{(1+z)^{2}+2 B z}\right]^{2}\right\} d z \\
= & \left(1+\frac{1}{c^{2}}\right) \frac{2 A^{2}}{\alpha} \int_{0}^{+\infty} \frac{z}{\left[(1+z)^{2}+2 B z\right]^{2}} d z \\
& +2 A^{2} \alpha \int_{0}^{+\infty} \frac{z\left(z^{2}-1\right)^{2}}{\left[(1+z)^{2}+2 B z\right]^{4}} d z .
\end{aligned}
$$

Substituting (67) into the above formula yields

$$
d^{\prime}(c)=\frac{6}{5} \frac{\left(c^{2}-1\right)^{3 / 2}\left(3 c^{2}+2\right)}{c^{3} b_{2}^{2}}
$$

Therefore

$$
d^{\prime \prime}(c)=\frac{18}{5} \frac{\sqrt{c^{2}-1}\left(2 c^{4}+c^{2}+2\right)}{c^{4} b_{2}^{2}} .
$$


We know that $2 c^{4}+c^{2}+2>0$ in (70). According to Theorem 7 , $\vec{\phi}_{c}$ is orbitally stable if $c>1$ and $b_{3}=0$. Thus, Corollary 12 holds.

According to the above Corollaries 11 and 12, we know that if $(4)$ has only one nonlinear term $b_{2}\left(u^{2}\right)_{x}$ or $b_{3}\left(u^{3}\right)_{x}$, that is, $b_{3}=0$ or $b_{2}=0$, the solitary waves of (4) are both orbitally stable if $c>1$. That is to say the wave speed intervals which make the two solitary waves stable are both $(1,+\infty)$. But according to Theorems 9 and 10, when (4) has two nonlinear items $b_{2}\left(u^{2}\right)_{x}$ and $b_{3}\left(u^{3}\right)_{x}$, the stability of solitary waves will be affected by the interaction between them. For convenience, we call the solitary wave whose wave speed $c$ satisfies $c>c_{0}$ $\left(c_{0}=\left(b_{2}^{2}+\sqrt{b_{2}^{4}+729 b_{3}^{2}}\right) / 27 b_{3}\right)$ the big wave speed solitary wave, while we call the solitary wave whose wave speed $c$ satisfies $c<c_{0}$ the small wave speed solitary wave. Generally, we have the results from Theorems 9 and 10 as follows.

(1) For given $b_{3}>0$, when $\left|b_{2}\right|$ is larger, the wave speed interval which makes the solitary waves stable will become smaller for the big wave speed solitary wave, but the wave speed interval which makes the solitary waves stable will become larger for the small wave speed solitary wave.

(2) For given $b_{2}$. For the big wave speed solitary wave, the wave speed interval which makes it stable will become larger if $b_{3}$ is bigger and the wave speed interval will become smaller if $b_{3}$ is smaller. For the small wave speed solitary wave, the wave speed interval which makes it stable will become smaller if $b_{3}$ is bigger and the wave speed interval will become larger if $b_{3}$ is smaller.

Summarizing the above results, it is significant to analyze the effect by multiple nonlinear terms on orbital stability of the solitary waves, at least in the application. For example, fix $b_{2}$ in (4). If we need to know the orbital stability of the small wave speed solitary wave in practical problems, since the wave speed interval which makes it stable will become larger as $b_{3}$ is smaller, and $\left(b_{2}^{2}+\sqrt{b_{2}^{4}+729 b_{3}^{2}}\right) / 27 b_{3} \rightarrow+\infty$ as $b_{3} \rightarrow 0$, it has little influence on the stability to ignore $b_{3} u^{3}$ in the application. But if we need to consider the orbital stability of the big wave speed solitary wave, the wave speed interval which makes it stable will become smaller as $b_{3}$ is smaller, so it is not suitable to ignore $b_{3} u^{3}$ in the application here.

\section{Instability of the Solitary Waves}

In this section, we will prove the conclusion (2) given in Theorem 7; that is, the solitary wave solution $\vec{\phi}_{c}$ is orbitally unstable if $d^{\prime \prime}(c)<0$.

Since $J$ given in Section 3 is not onto, we cannot apply Grillakis-Shatah-Strauss theory on the system (4) directly. In order to prove instability, we define a new conservational functional

$$
I(\vec{u})=\int_{-\infty}^{\infty} \vec{u}(x) d x .
$$

We will prove that $d^{\prime \prime}(c)<0$ is the sufficient condition to judge orbital instability of solitary wave solutions by estimating to the solution of initial value problem.

\subsection{Estimate to the Solution of Initial Value Problem for (4)}

Lemma 13. The unique solution $\vec{u}(t)$ of (4) with initial data $\vec{u}(0)=\vec{u}_{0}$ satisfies

$$
\begin{array}{ll}
E(\vec{u}(t))=E(u, v)=\text { constant }, & t \in R^{+}, \\
V(\vec{u}(t)) & =V(u, v)=\text { constant }, \quad t \in R^{+},
\end{array}
$$

where $E(\vec{u})=-\int_{R}\left(v u+\left(b_{2} / 3\right) u^{3}+\left(b_{3} / 4\right) u^{4}\right) d x$ and $V(\vec{u})=$ $(1 / 2) \int_{R}\left(-u_{x x} u+u^{2}+v^{2}\right) d x$.

From Lemma 4, (23), and (27), we can prove Lemma 13 easily. We now prove that $I(\vec{u})=\int_{-\infty}^{\infty} \vec{u}(x) d x$ is an invariance.

Lemma 14. If $\int_{R} u_{0}(x) d x$ and $\int_{R} v_{0}(x) d x$ converge, then $I(u)=\int_{R} u d x$ and $I(v)=\int_{R} v d x$ converge and are constants for any $t \in R^{+}$.

Proof. Integrating (4) separately yields

$$
\begin{aligned}
& \int_{a}^{b} u(x, t) d x-\int_{a}^{b} u(x, 0) d x \\
& \quad=\int_{0}^{t} \int_{a}^{b} \frac{\partial}{\partial x}\left(\frac{\partial}{\partial x^{2}}-1\right)^{-1}\left(v+b_{2} u^{2}+b_{3} u^{3}\right) d x d \tau \\
& \int_{a}^{b} v(x, t) d x-\int_{a}^{b} v(x, 0) d x=-\int_{0}^{t} \int_{a}^{b} \frac{\partial}{\partial x} u(x, \tau) d x d \tau .
\end{aligned}
$$

Now we analyze the second formula and have

$$
-\int_{0}^{t} \int_{a}^{b} u_{x} d x d \tau=-\int_{0}^{t}[u(b, \tau)-u(a, \tau)] d \tau .
$$

For any fixed $\tau, u(b, \tau) \rightarrow 0$ and $u(a, \tau) \rightarrow 0$, as $a \rightarrow-\infty$ and $b \rightarrow+\infty$,

$$
-\int_{a}^{b} \int_{0}^{t} u_{x} d \tau d x \longrightarrow 0
$$

Thus

$$
\int_{a}^{b} v(x, t) d x-\int_{a}^{b} v(x, 0) d x \longrightarrow 0
$$

Similarly,

$$
\int_{a}^{b} u(x, t) d x-\int_{a}^{b} u(x, 0) d x \longrightarrow 0 .
$$

Hence $I(u)=\int_{R} u d x$ and $I(v)=\int_{R} v d x$ exist and are equal to $\int_{R} u_{0}(x) d x$ and $\int_{R} v_{0}(x) d x$, respectively. This completes the proof of Lemma 14.

The next theorem is the key step in the proof of instability, and it is the main result of this section. 
Theorem 15. Let $\Lambda^{1} u_{0} \in L^{1}$ and $v_{0} \in L^{1}$, where $\Lambda^{k}=$ $\left(1-\partial^{2} / \partial x^{2}\right)^{k / 2}(k \in Z)$. Assume that $\vec{u}=\left(\begin{array}{l}u \\ v\end{array}\right)$ satisfies (4) and $u(0, x)=u_{0}$. Then

$$
\sup _{-\infty<x<\infty}\left|\int_{-\infty}^{x} \vec{u}(z, t) d z\right| \leq c_{0}\left(1+t^{2 / 3}+t^{9 / 10}\right)
$$

where the constant $c_{0}$ only depends on $\vec{u}_{0}$.

In order to prove Theorem 15, we need a series of lemmas. The first one is the well-known Van der Corput lemma [12]. The proofs of the following Lemmas 17 and 18 are similar to those which are given in [13], and we omit the details.

Lemma 16 (Van der Corput lemma). Let $h(\xi)$ be either convex or concave on $[a, b]$ with $-\infty \leq a<b \leq \infty$. If $h^{\prime \prime}(\xi) \neq 0$ in $[a, b]$; then

$$
\left|\int_{a}^{b} e^{i h(\xi)} d \xi\right| \leq 4\left\{\min _{[a, b]}\left|h^{\prime \prime}(\xi)\right|\right\}^{-1 / 2}
$$

Lemma 17. Suppose $t>0, n>0$, one has

$$
\sup _{-\infty<\alpha<+\infty}\left|\int_{-n}^{n} e^{i t h(\xi, \alpha)} d \xi\right| \leq c_{0}\left(t^{-1 / 3}+t^{-1 / 2} n^{2}\right)
$$

where $c_{0}$ is a constant and $h(\xi, \alpha)=\xi / \sqrt{1+\xi^{2}}+\alpha \xi$.

Lemma 18. For $1 \leq p \leq \infty$, if $u \in L^{p}(R)$, we have $\Lambda^{-1} u \in L^{p}$ and $\left\|\Lambda^{-1} u\right\|_{p} \leq\|u\|_{p}$.

The following lemma concerns the decay of the linear evolution operator.

Lemma 19. Suppose that $S(t)$ the evolution operator of the linear equation

$$
\vec{u}_{t}+\left(\begin{array}{cc}
0 & (1-\Delta)^{-1} \\
1 & 0
\end{array}\right) \vec{u}_{x}=0, \quad \vec{u}(0)=\vec{u}_{0}=\left(\begin{array}{l}
u_{0} \\
v_{0}
\end{array}\right) .
$$

That is to say, $S(t) \vec{u}(0)=\vec{u}(t)$. If $\Lambda^{1} u_{0} \in L^{1}$ and $v_{0} \in L^{1}$, we have $S(t) \vec{u}_{0} \in L^{\infty}$ and

$$
\left\|S(t) \vec{u}_{0}\right\|_{\infty} \leq c_{0}\left(t^{-1 / 3}+t^{-1 / 10}\right)\left(\left\|\Lambda^{1} u_{0}\right\|_{1}+\left\|v_{0}\right\|_{1}\right),
$$

where $c_{0}$ is a constant.

Proof. The solution of the linear equation is

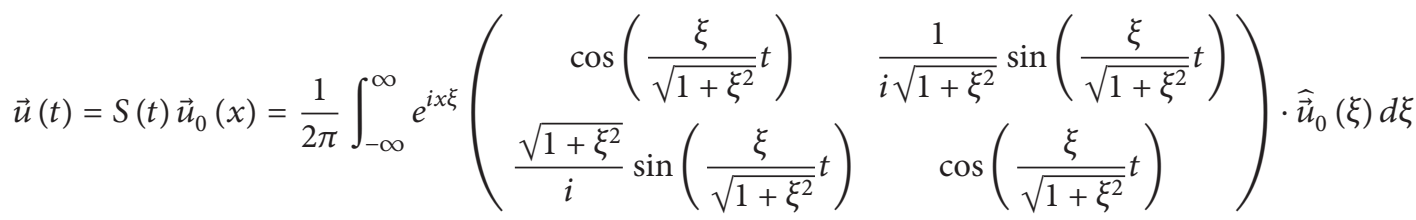

$$
\begin{aligned}
& =\frac{1}{2 \pi} \int_{-\infty}^{\infty} e^{i x \xi}\left(\begin{array}{cc}
\cos \left(-\frac{\xi}{\sqrt{1+\xi^{2}}} t\right) & \frac{i}{\sqrt{1+\xi^{2}}} \sin \left(-\frac{\xi}{\sqrt{1+\xi^{2}}} t\right) \\
i \sqrt{1+\xi^{2}} \sin \left(-\frac{\xi}{\sqrt{1+\xi^{2}}} t\right) & \cos \left(-\frac{\xi}{\sqrt{1+\xi^{2}}} t\right)
\end{array}\right) \cdot \widehat{\vec{u}}_{0}(\xi) d \xi,
\end{aligned}
$$

where $\widehat{\vec{u}}_{0}$ is the Fourier transform of $\vec{u}_{0}$.

According to Fubini's theorem and Lemmas 17 and 18, we have

$$
\begin{aligned}
& |\vec{u}(t)| \\
& =\left|S(t) \vec{u}_{0}(x)\right| \\
& \leq \frac{1}{4 \pi} \sum\left|\int_{-\infty}^{+\infty}\left(\widehat{u}_{0} \pm \frac{1}{\sqrt{1+\xi^{2}}} \widehat{v}_{0}\right) e^{i t\left(-\xi / \sqrt{1+\xi^{2}} \pm(x / t) \xi\right)} d \xi\right| \\
& \quad+\frac{1}{4 \pi} \sum\left|\int_{-\infty}^{+\infty}\left(\widehat{v}_{0} \pm \sqrt{1+\xi^{2}} \widehat{u}_{0}\right) e^{i t\left(-\xi / \sqrt{1+\xi^{2}} \pm(x / t) \xi\right)} d \xi\right| \\
& \leq \frac{1}{4 \pi} \sum\left|\int_{-\infty}^{+\infty}\left(\widehat{u}_{0} \pm \widehat{\Lambda^{-1} v_{0}}\right) e^{i t\left(-\xi / \sqrt{1+\xi^{2}} \pm(x / t) \xi\right)} d \xi\right|
\end{aligned}
$$

$$
\begin{aligned}
& +\frac{1}{4 \pi} \sum\left|\int_{-\infty}^{+\infty}\left(\widehat{v}_{0} \pm \widehat{\Lambda^{1} u_{0}}\right) e^{i t\left(-\xi / \sqrt{1+\xi^{2}} \pm(x / t) \xi\right)} d \xi\right| \\
& \leq \frac{1}{2 \pi} \int_{|\xi|>n}\left(\left|\widehat{u}_{0}\right|+\left|\widehat{\Lambda^{-1} v_{0}}\right|+\left|\widehat{v}_{0}\right|+\left|\widehat{\Lambda^{1} u_{0}}\right|\right) d \xi \\
& +\frac{1}{4 \pi} \sum \int_{R}\left|u_{0}(y) \pm \Lambda^{-1} v_{0}(y)\right| \\
& \quad \times\left|\int_{-n}^{n} e^{i t\left(-\xi / \sqrt{1+\xi^{2}} \pm(x / t) \xi-(y / t) \xi\right)} d \xi\right| d y \\
& +\frac{1}{4 \pi} \sum \int_{R}\left|v_{0}(y) \pm \Lambda^{1} u_{0}(y)\right| \\
& \quad \times\left|\int_{-n}^{n} e^{i t\left(-\xi / \sqrt{1+\xi^{2}} \pm(x / t) \xi-(y / t) \xi\right)} d \xi\right| d y .
\end{aligned}
$$


Therefore,

$$
\begin{aligned}
|\vec{u}(t)| \leq & c_{0}\left(\left\|\Lambda^{1} u_{0}\right\|_{L^{1}}+\left\|v_{0}\right\|_{L^{1}}\right) \\
& \times\left(\int_{|\xi|>n}(1+|\xi|)^{-2} d \xi\right)^{1 / 2} \\
& +c_{0}\left(\left\|\vec{u}_{0}\right\|_{L^{1} \times L^{1}}+\left\|\Lambda^{-1} v_{0}\right\|_{L^{1}}+\left\|\Lambda^{1} u_{0}\right\|_{L^{1}}\right) \\
& \times \sup _{-\infty<\alpha<\infty}\left|\int_{-\infty}^{\infty} e^{i t h(\xi, \alpha)} d \xi\right| \\
\leq & c_{0}\left(n^{-1 / 2}+t^{-1 / 3}+t^{-1 / 2} n^{2}\right)\left(\left\|v_{0}\right\|_{L^{1}}+\left\|\Lambda^{1} u_{0}\right\|_{L^{1}}\right) .
\end{aligned}
$$

Choosing $n=t^{1 / 5}$, we have

$$
\left|S(t) \vec{u}_{0}\right| \leq c_{0}\left(t^{-1 / 3}+t^{-1 / 10}\right)\left(\left\|v_{0}\right\|_{L^{1}}+\left\|\Lambda^{1} u_{0}\right\|_{L^{1}}\right),
$$

where $t>0$. This completes the proof of Lemma 19 .

Proof of Theorem 15. Let $\vec{w}(t)=S(t) \vec{u}_{0}$. Then $\vec{w}(t)$ satisfies

$$
\vec{w}_{t}+\left(\begin{array}{c}
0 \\
1
\end{array}\left(\begin{array}{c}
1-\frac{\partial^{2}}{\partial x^{2}} \\
0
\end{array}\right) \vec{w}_{x}=0, \quad \text { with } \vec{w}(0)=\vec{u}_{0} .\right.
$$

The solution $\vec{u}(t)$ of the nonlinear (4) can be written as

$$
\vec{u}(t)=\vec{w}(t)+\frac{\partial}{\partial x} \int_{0}^{t} S(t-\tau)\left(\begin{array}{c}
-\Lambda^{-2}\left(b_{2} u^{2}+b_{3} u^{3}\right) \\
0
\end{array}\right) d \tau .
$$

Let $\vec{U}(x, t)=\int_{-\infty}^{x} \vec{u}(y, t) d y, \vec{U}(x, 0)=\int_{-\infty}^{x} \vec{u}(y, 0) d y$, and $\vec{W}(x, t)=\int_{-\infty}^{x} \vec{w}(y, t) d y$. Then $\vec{U}(x, t)=\vec{W}(t)-\int_{0}^{t} S(t-$ $\tau)\left(\begin{array}{c}\Lambda^{-2}\left(b_{2} u^{2}+b_{3} u^{3}\right) \\ 0\end{array}\right) d \tau$.

We estimate both two terms in the above formula on the right-hand side separately. Firstly, from the equation for $\vec{w}(t)$, we can obtain

$$
\vec{w}(t)=\vec{u}_{0}-\partial_{x} \int_{0}^{t}\left(\begin{array}{cc}
0 & (1-\Delta)^{-1} \\
1 & 0
\end{array}\right) \vec{w}(\tau) d \tau .
$$

Therefore

$$
\vec{W}(t)=\vec{U}_{0}-\int_{0}^{t}\left(\begin{array}{cc}
0 & (1-\Delta)^{-1} \\
1 & 0
\end{array}\right) \vec{w}(\tau) d \tau .
$$

Since $\vec{w}(t)=S(t) \vec{u}_{0}$, we obtain

$$
\begin{aligned}
& |\vec{W}(x, t)| \\
& \quad \leq\left|\vec{u}_{0}\right|_{L^{1} \times L^{1}}+\left|\int_{0}^{t}\left(\begin{array}{cc}
0 & (1-\Delta)^{-1} \\
1 & 0
\end{array}\right) S(\tau)\left(\begin{array}{l}
u_{0} \\
v_{0}
\end{array}\right) d \tau\right| \\
& \quad=\left|\vec{u}_{0}\right|_{L^{1} \times L^{1}}+\left|\int_{0}^{t} S(\tau)\left(\begin{array}{c}
(1-\Delta)^{-1} v_{0} \\
u_{0}
\end{array}\right) d \tau\right| \\
& \quad \leq\left|\vec{u}_{0}\right|_{L^{1} \times L^{1}}+\int_{0}^{t}\left|S(\tau)\left(\begin{array}{c}
(1-\Delta)^{-1} v_{0} \\
u_{0}
\end{array}\right)\right| d \tau .
\end{aligned}
$$

Using Lemma 19, substituting $\left(\begin{array}{c}(1-\Delta)^{-1} v_{0} \\ u_{0}\end{array}\right)$ for $\left(\begin{array}{c}u_{0} \\ v_{0}\end{array}\right)$, we have

$$
\begin{aligned}
|\vec{W}(x, t)| \leq & \left|\vec{u}_{0}\right|_{L^{1} \times L^{1}} \\
& +c_{0} \int_{0}^{t}\left(\tau^{-1 / 3}+\tau^{-1 / 10}\right) d \tau\left(\left\|\Lambda^{-1} v_{0}\right\|_{L^{1}}+\left\|u_{0}\right\|_{L^{1}}\right) \\
\leq & c_{0}\left(1+t^{2 / 3}+t^{9 / 10}\right)\left(\left\|v_{0}\right\|_{L^{1}}+\left\|u_{0}\right\|_{L^{1}}\right) .
\end{aligned}
$$

Let

$$
\vec{Y}(x, t)=\int_{0}^{t} S(t-\tau)\left(\begin{array}{c}
\Lambda^{-2}\left(b_{2} u^{2}+b_{3} u^{3}\right) \\
0
\end{array}\right) d \tau .
$$

Using Lemma 19 again, and substituting $\left(\begin{array}{c}\Lambda^{-2}\left(b_{2} u^{2}+b_{3} u^{3}\right) \\ 0\end{array}\right)$ for $\left(\begin{array}{l}u_{0} \\ v_{0}\end{array}\right)$, we obtain

$$
\begin{aligned}
& |\vec{Y}(x, t)| \\
& \leq \int_{0}^{t}\left|S(t-\tau)\left(\begin{array}{c}
\Lambda^{-2}\left(b_{2} u^{2}+b_{3} u^{3}\right) \\
0
\end{array}\right)\right| d \tau \\
& \leq c_{0} \int_{0}^{t}\left((t-\tau)^{-1 / 3}+(t-\tau)^{-1 / 10}\right)\left\|\Lambda^{-1}\left(b_{2} u^{2}+b_{3} u^{3}\right)\right\|_{L^{1}} d \tau .
\end{aligned}
$$

In view of Lemma 18 , we have

$$
\left\|\Lambda^{-1}\left(b_{2} u^{2}+b_{3} u^{3}\right)\right\|_{L^{1}} \leq\left\|b_{2} u^{2}+b_{3} u^{3}\right\|_{L^{1}} .
$$

Therefore

$$
\begin{aligned}
& |\vec{Y}(x, t)| \\
& \leq c_{0} \int_{0}^{t}\left((t-\tau)^{-1 / 3}+(t-\tau)^{-1 / 10}\right)\left(\left\|u^{2}\right\|_{L^{1}}+\left\|u^{3}\right\|_{L^{1}}\right) d \tau \\
& \leq c_{0}\left(t^{2 / 3}+t^{9 / 10}\right) .
\end{aligned}
$$

Summarizing the estimate of $\vec{W}(x, t)$ and $\vec{Y}(x, t)$ above yields the result of Theorem 15; that is,

$$
\sup _{-\infty<x<\infty}\left|\int_{-\infty}^{x} \vec{u}(z, t) d z\right| \leq c_{0}\left(1+t^{2 / 3}+t^{9 / 10}\right) .
$$

\subsection{Proof of Instability}

Theorem 20. Let $c \neq 0$ be fixed. If $d^{\prime \prime}(c)<0$, then there is a curve $\omega \rightarrow \Phi_{\omega}$ such that $V\left(\vec{\Phi}_{\omega}\right)=V\left(\vec{\varphi}_{c}\right), \vec{\Phi}_{c}=\vec{\varphi}_{c}$, and on which $E(\vec{u})$ has a strict local maximum at $\vec{u}=\vec{\varphi}_{c}$.

Proof. Let $\chi_{c}$ be the unique negative eigenfunction of $H_{c}$, which has been proved in Section 2. Next we define

$$
\vec{\Phi}_{\omega}=\vec{\varphi}_{\omega}+s(\omega) \vec{\chi}_{c}, \quad \text { for } \omega \longrightarrow c
$$

where $s(\omega)$ satisfies $s(c)=0$ and $V\left(\vec{\Phi}_{\omega}\right)=V\left(\vec{\varphi}_{c}\right)$. 
By the implicit function theorem, the function $s(\omega)$ can be determined. In fact,

$$
\begin{aligned}
& \left.\frac{\partial}{\partial s} V\left(\vec{\varphi}_{\omega}+s \vec{\chi}\right)\right|_{\{s=0, \omega=c\}} \\
& \quad=\left\langle V^{\prime}\left(\vec{\varphi}_{c}\right), \vec{\chi}_{c}\right\rangle=\int_{-\infty}^{\infty}\left(\Lambda^{2} \varphi_{c} \cdot \chi_{1, c}+\psi_{c} \cdot \chi_{2, c}\right) d x,
\end{aligned}
$$

where $\chi_{c}=\left(\begin{array}{l}\chi_{1, c} \\ \chi_{2, c}\end{array}\right)$ with $\chi_{1, c}=(c-\lambda) \chi_{2, c}$, and $\lambda$ is the unique negative eigenvalue of $H_{c}$ and $\varphi_{c}=c \psi_{c}, \varphi_{c}>0, \chi_{1, c}>$ $0, \Lambda^{2} \varphi_{c}>0$. Therefore

$$
\begin{aligned}
\frac{\partial}{\partial s} V & \left.\left(\vec{\varphi}_{\omega}+s \vec{\chi}\right)\right|_{\{s=0, \omega=c\}} \\
& =\int_{-\infty}^{+\infty}\left(\Lambda^{2} \varphi_{c} \cdot \chi_{1, c}+\psi_{c} \cdot \chi_{2, c}\right) d x \\
& =\int_{-\infty}^{+\infty}\left(\Lambda^{2} \varphi_{c} \cdot \chi_{1, c}+\frac{1}{c(c-\lambda)} \varphi_{c} \cdot \chi_{1, c}\right) d x \neq 0 .
\end{aligned}
$$

It is easy to see that

$$
\begin{array}{r}
\qquad\left.\frac{d^{2}}{d \omega^{2}} E\left(\vec{\Phi}_{\omega}\right)\right|_{\omega=c}=\left\langle H_{c} \vec{y}, \vec{y}\right\rangle, \\
\text { where } \vec{y}=\left.\frac{\partial \vec{\Phi}_{\omega}}{\partial \omega}\right|_{\omega=c}=\frac{\partial \vec{\varphi}_{c}}{\partial c}+s^{\prime}(c) \vec{\chi}_{c} .
\end{array}
$$

So it suffices to show that $\left\langle H_{c} \vec{y}, \vec{y}\right\rangle<0$. Since

$$
\begin{aligned}
0 & =\frac{d V\left(\vec{\varphi}_{c}\right)}{d \omega}=\frac{\left.d V\left(\vec{\Phi}_{\omega}\right)\right|_{\omega=c}}{d \omega} \\
& =\left\langle V^{\prime}\left(\vec{\varphi}_{c}\right), \vec{y}\right\rangle=\left.\left\langle V^{\prime}\left(\vec{\varphi}_{\omega}\right), \vec{y}\right\rangle\right|_{\omega=c^{\prime}}
\end{aligned}
$$

then

$$
\begin{aligned}
d^{\prime \prime}(c) & =\left\langle V^{\prime}\left(\vec{\varphi}_{c}\right), \frac{d \vec{\varphi}_{c}}{d c}\right\rangle=\left\langle V^{\prime}\left(\vec{\varphi}_{c}\right), \vec{y}-s^{\prime}(c) \vec{\chi}_{c}\right\rangle \\
& =\left\langle V^{\prime}\left(\vec{\varphi}_{c}\right), \vec{y}\right\rangle-\left\langle V^{\prime}\left(\vec{\varphi}_{c}\right), s^{\prime}(c) \vec{\chi}_{c}\right\rangle \\
& =-s^{\prime}(c)\left\langle V^{\prime}\left(\vec{\varphi}_{c}\right), \vec{\chi}_{c}\right\rangle, \\
H_{c} \vec{y} & =H_{c} \frac{\partial \vec{\varphi}_{c}}{\partial c}+H_{c} s^{\prime}(c) \vec{\chi}_{c} \\
& =\left(E^{\prime \prime}\left(\vec{\varphi}_{c}\right)+c V^{\prime \prime}\left(\vec{\varphi}_{c}\right)\right) \frac{\partial \vec{\varphi}_{c}}{\partial c}+H_{c} s^{\prime}(c) \vec{\chi}_{c} .
\end{aligned}
$$

Note that $E^{\prime}\left(\vec{\varphi}_{c}\right)+c V^{\prime}\left(\vec{\varphi}_{c}\right)=0$. We derivate it with respect to $c$, and then

$$
E^{\prime \prime}\left(\vec{\varphi}_{c}\right) \frac{\partial \vec{\varphi}_{c}}{\partial c}+V^{\prime}\left(\vec{\varphi}_{c}\right)+c V^{\prime \prime}\left(\vec{\varphi}_{c}\right) \frac{\partial \vec{\varphi}_{c}}{\partial c}=0 .
$$

Namely,

$$
\left(E^{\prime \prime}\left(\vec{\varphi}_{c}\right)+c V^{\prime \prime}\left(\vec{\varphi}_{c}\right)\right) \frac{\partial \vec{\varphi}_{c}}{\partial c}+V^{\prime}\left(\vec{\varphi}_{c}\right)=0 .
$$

So

$$
\begin{gathered}
H_{c} \vec{y}=-V^{\prime}\left(\vec{\varphi}_{c}\right)+s^{\prime}(c) H_{c} \vec{\chi}_{c}, \\
\left\langle H_{c} \vec{y}, \vec{y}\right\rangle \\
=\left\langle-V^{\prime}\left(\vec{\varphi}_{c}\right)+s^{\prime}(c) H_{c} \vec{\chi}_{c}, \frac{\partial \vec{\varphi}_{c}}{\partial c}+s^{\prime}(c) \vec{\chi}_{c}\right\rangle \\
=\left\langle-V^{\prime}\left(\vec{\varphi}_{c}\right), \frac{\partial \vec{\varphi}_{c}}{\partial c}\right\rangle+\left\langle-V^{\prime}\left(\vec{\varphi}_{c}\right), s^{\prime}(c) \vec{\chi}_{c}\right\rangle \\
+\left\langle s^{\prime}(c) H_{c} \vec{\chi}_{c}, \frac{\partial \vec{\varphi}_{c}}{\partial c}\right\rangle+\left\langle s^{\prime}(c) H_{c} \vec{\chi}_{c}, s^{\prime}(c) \vec{\chi}_{c}\right\rangle \\
=s^{\prime}(c)\left\langle H_{c} \vec{\chi}_{c}, \frac{\partial \vec{\varphi}_{c}}{\partial c}\right\rangle+\left(s^{\prime}(c)\right)^{2}\left\langle H_{c} \vec{\chi}_{c}, \vec{\chi}_{c}\right\rangle \\
=s^{\prime}(c)\left\langle H_{c} \frac{\partial \vec{\varphi}_{c}}{\partial c}, \vec{\chi}_{c}\right\rangle+\left(s^{\prime}(c)\right)^{2}\left\langle H_{c} \vec{\chi}_{c}, \vec{\chi}_{c}\right\rangle \\
=s^{\prime}(c)\left\langle-V^{\prime}\left(\vec{\varphi}_{c}\right), \vec{\chi}_{c}\right\rangle+\left(s^{\prime}(c)\right)^{2}\left\langle H_{c} \vec{\chi}_{c}, \vec{\chi}_{c}\right\rangle \\
=d^{\prime \prime}(c)+\left(s^{\prime}(c)\right)^{2}\left\langle H_{c} \vec{\chi}_{c}, \vec{\chi}_{c}\right\rangle\langle 0 .
\end{gathered}
$$

Hence, $\left.\left(d^{2} / d \omega^{2}\right) E\left(\vec{\Phi}_{\omega}\right)\right|_{\omega=c}=\left\langle H_{c} \vec{y}, \vec{y}\right\rangle<0$. The result in Theorem 20 holds.

Lemma 21 (see [14]). There exists $\varepsilon>0$ and a unique $C^{1}$ map $\alpha: U_{\varepsilon} \rightarrow R$, such that for any $\vec{u} \in U_{\varepsilon}$ and $r \in R$,

$$
\begin{aligned}
& \text { (1) }\left\langle\vec{u}(\cdot+\alpha(\vec{u})), T^{\prime}(0) \vec{\varphi}_{c}\right\rangle=0, \\
& \text { (2) } \alpha(\vec{u}(\cdot+r))=\alpha(\vec{u})-r \\
& \text { (3) } \alpha^{\prime}(\vec{u})=\frac{(\partial / \partial x) \vec{\varphi}_{c}(\cdot-\alpha(\vec{u}))}{\left\langle\vec{u},\left(\partial^{2} / \partial x^{2}\right) \vec{\varphi}_{c}(\cdot-\alpha(\vec{u}))\right\rangle},
\end{aligned}
$$

where

$$
U_{\varepsilon}=\left\{\vec{u} \in X: \inf _{s \in R}\left\|\vec{u}-\tau_{s} \vec{\varphi}_{c}\right\|_{X}<\varepsilon\right\} .
$$

Next we define an auxiliary operator $B$ which will play a critical role in the proof of instability.

Definition 22. For $\vec{u} \in U_{\varepsilon}, B(\vec{u})$ is defined by the formula

$$
B(\vec{u})=\vec{y}(\cdot-\alpha(\vec{u}))-\langle I \vec{u}, \vec{y}(\cdot-\alpha(\vec{u}))\rangle \frac{\partial}{\partial x} I^{-1} \alpha^{\prime}(\vec{u}) .
$$

By Lemma 21, $B(\vec{u})$ can also be written as

$$
\begin{aligned}
B(\vec{u})= & \vec{y}(\cdot-\alpha(\vec{u})) \\
& -\frac{\langle I \vec{u}, \vec{y}(\cdot-\alpha(\vec{u}))\rangle}{\left\langle\vec{u},\left(\partial^{2} / \partial x^{2}\right) \vec{\varphi}_{c}(\cdot-\alpha(\vec{u}))\right\rangle} I^{-1} \frac{\partial^{2}}{\partial x^{2}} \varphi_{c}(\cdot-\alpha(\vec{u})),
\end{aligned}
$$

where $I=\left(\begin{array}{cc}1-\Delta & 0 \\ 0 & 1\end{array}\right)$.

The next lemma summarizes the properties of $B$. 
Lemma 23 (see [14]). $B(\vec{u}): U_{\varepsilon} \rightarrow X$ is a $C^{1}$ function. Moreover, $B$ commutes with translations, $B\left(\vec{\varphi}_{c}\right)=\vec{y}$ and for any $\vec{u} \in U_{\varepsilon},\langle B(\vec{u}), I \vec{u}\rangle=0$.

Lemma 24 (see [14]). There exists a $C^{1}$ function

$$
\Pi:\left\{\vec{v} \in U_{\varepsilon}: V(\vec{v})=V\left(\vec{\varphi}_{c}\right)\right\} \longrightarrow R,
$$

which is invariant under translations, such that

$$
E\left(\vec{\varphi}_{c}\right)<E(\vec{v})+\Pi(\vec{v})\left\langle E^{\prime}(\vec{v}), B(\vec{v})\right\rangle,
$$

for any $\vec{v} \in U_{\varepsilon}$ with $V(\vec{v})=V\left(\vec{\varphi}_{c}\right)$ and $\vec{v}$ is not a translate of $\vec{\varphi}_{c}$.

Lemma 25 (see [14]). According to Theorem 20, there is a curve $\omega \rightarrow \Phi_{\omega}$ which satisfies $E\left(\vec{\Phi}_{\omega}\right)<E\left(\vec{\varphi}_{c}\right)$ for $\omega \neq c$, $V\left(\vec{\Phi}_{\omega}\right)=V\left(\vec{\varphi}_{c}\right)$, and $\left\langle E^{\prime}\left(\vec{\Phi}_{\omega}\right), B\left(\vec{\Phi}_{\omega}\right)\right\rangle$ changes sign as $\omega$ passes through $c$, with $c \neq 0$.

Theorem 26. If (4) has a bell-profile solitary wave solution $\vec{\phi}_{c}$, when $d^{\prime \prime}(c)<0$, then $\vec{\phi}_{c}$ is orbitally unstable.

Proof. Firstly, we consider $c \neq 0$. Let $\varepsilon>0$, small enough, and $U_{\varepsilon}$ be the tubular neighbourhood defined above. By Lemma 25 we can choose $\vec{u}_{0} \in X$ which is arbitrarily close to $\vec{\varphi}_{c}$, such that $V\left(\vec{u}_{0}\right)=V\left(\vec{\varphi}_{c}\right), E\left(\vec{u}_{0}\right)<E\left(\vec{\varphi}_{c}\right)$, and $\left\langle E^{\prime}\left(\overrightarrow{\mathcal{u}}_{0}\right), B\left(\overrightarrow{\mathcal{u}}_{0}\right)\right\rangle>0$. To prove the instability of $\vec{\varphi}_{c}$, it suffices to show that there are some elements $\vec{u}_{0} \in X$ which are arbitrarily close to $\vec{\varphi}_{c}$, but the solution $\vec{u}(x, t)$ with the initial data $\vec{u}_{0}$ exits from $U_{\varepsilon}$ in finite time. Let $\left[0, t_{1}\right)$ be the maximal interval for which $\vec{u}(x, t)$ lies continuously in $U_{\varepsilon}$, where $t_{1}>$ 0 . Let $T$ be the maximum existence time for the solution $\vec{u}(x, t)$ with initial data $\vec{u}_{0}$. If $T$ is finite, it is easy to see that $\vec{\varphi}_{c}$ is orbital instability by definition, so we may assume that $T=+\infty$ and our purpose now is to show that $t_{1}<+\infty$; that is to say, it is instability if it blows up at a finite time. The proof is as follows.

Firstly, in view of Lemmas 4, 13, and 14 and Theorem 15, we know that $\vec{u}$ enjoys the following properties:

$$
\vec{u} \in C\left(\left[0, t_{1}\right) ; X\right), \quad \vec{u}(0, x)=\vec{u}_{0}(x),
$$

$E(\vec{u}(t)), V(\vec{u}(t))$ are constant, for $t \in\left[0, t_{1}\right)$,

$I(u(t)), I(v(t))$ converge and are constant,

$$
\text { for } t \in\left[0, t_{1}\right) \text {, }
$$

$$
\sup _{0 \leq t<t_{1}}\|\vec{u}(x, t)\|_{X} \leq c_{1},
$$

$$
\sup _{x \in R}\left|\int_{-\infty}^{x} \vec{u}(y, t) d y\right| \leq c_{2}\left(1+t^{2 / 3}+t^{9 / 10}\right)
$$

where $c_{1}$ depends on $\vec{\varphi}_{c}$ and $\varepsilon, c_{2}$ depends on $c_{1},\left\|v_{0}\right\|_{L^{1}}$, and $\left\|\vec{u}_{0}\right\|_{L^{1}}$.
Let $\beta(t)=\alpha(\vec{u}(t))$, where $\alpha$ is defined by Lemma 21 and define

$$
\begin{gathered}
\vec{y}(z)=\frac{d \vec{\Phi}_{c}}{d c}=\frac{d \vec{\varphi}_{c}}{d c}+s^{\prime}(c) \vec{\chi}_{c}, \\
\vec{Y}(x)=\int_{-\infty}^{x} I \vec{y}(z) d z, \\
A(t)=\int_{-\infty}^{\infty} \vec{Y}(x-\beta(t)) \cdot \vec{u}(x, t) d x, \quad 0 \leq t<t_{1},
\end{gathered}
$$

where the function $A(t)$ serves as a Lyapunov function, and

$$
\vec{\gamma}=\int_{-\infty}^{\infty} \vec{y}(x) d x
$$

Due to the assumptions above, it is observed that

$$
\begin{gathered}
\int_{-\infty}^{\infty}(1+|x|)^{1 / 2} \frac{\partial \vec{\varphi}_{c}}{\partial c} d x<\infty \\
\int_{-\infty}^{\infty}(1+|x|)^{1 / 2} \vec{\chi}_{c} d x<\infty
\end{gathered}
$$

Therefore $\int_{-\infty}^{\infty}(1+|x|)^{1 / 2}|\vec{y}(x)| d x<\infty$, such that $|\vec{\gamma}|<+\infty$. Indeed, if $H$ is the Heaviside function and $\vec{W}(x)=$ $\int_{-\infty}^{x} \vec{y}(z) d z-\vec{\gamma} H(x)$, then

$$
\begin{array}{cl}
\vec{W}(x)=\int_{-\infty}^{x} \vec{y}(z) d z & \text { for } x<0, \\
\vec{W}(x)=-\int_{x}^{\infty} \vec{y}(z) d z & \text { for } x \geq 0 .
\end{array}
$$

Now we can have

$$
\begin{aligned}
A(t)= & \int_{-\infty}^{\infty}(\vec{Y}(x-\beta(t))-\vec{\gamma} H(x-\beta(t))) \cdot \vec{u}(x, t) d x \\
& +\vec{\gamma} \int_{-\infty}^{\infty} \vec{H}(x-\beta(t)) \cdot \vec{u}(x, t) d x \\
= & \int_{-\infty}^{\infty}(\vec{Y}(x-\beta(t))-\vec{\gamma} H(x-\beta(t))) \cdot \vec{u}(x, t) d x \\
& +\vec{\gamma} \int_{\beta(t)}^{\infty} \vec{u}(x, t) d x .
\end{aligned}
$$

Hence, by (113),

$$
|A(t)| \leq\|\vec{Y}-\vec{\gamma} H\|_{0}\|\vec{u}(t)\|_{0}+c_{2}\left(1+t^{2 / 3}+t^{9 / 10}\right) .
$$


It follows from Minkowski’s inequality that

$$
\begin{aligned}
& \left(\int_{-\infty}^{0}|\vec{Y}(x)-\vec{\gamma} H(x)|^{2} d x\right)^{1 / 2} \\
& \quad=\left(\int_{-\infty}^{0}|\vec{Y}(x)|^{2} d x\right)^{1 / 2} \\
& \quad \leq \int_{-\infty}^{0}\left(\int_{z}^{0}|I \vec{y}(z)|^{2} d x\right)^{1 / 2} d z \\
& \quad=\int_{-\infty}^{0}\left(\int_{z}^{0}|\vec{y}(z)|^{2} d x\right)^{1 / 2} d z \\
& \quad=\int_{-\infty}^{0}|\vec{y}(z)| \sqrt{-z} d z<\infty .
\end{aligned}
$$

Similarly, $\left(\int_{0}^{\infty}|\vec{Y}-\vec{\gamma} H(x)|^{2} d x\right)^{1 / 2}<\infty$. Therefore

$$
|A(t)| \leq c_{2}\left(1+t^{2 / 3}+t^{9 / 10}\right), \quad \text { for } 0 \leq t<t_{1} .
$$

Since $A(t)=\langle\vec{Y}(x-\beta(t)), \vec{u}(x, t)\rangle$, and $\beta(t)=\alpha(\vec{u}(t))$, now we estimate $d A(t) / d t$, by calculating, and have

$$
\begin{aligned}
& \frac{d A(t)}{d t} \\
& =\left\langle\frac{d \vec{Y}(x-\beta(t))}{d t}, \vec{u}(x, t)\right\rangle+\left\langle\vec{Y}(x-\beta(t)), \frac{\partial}{\partial t} \vec{u}(x, t)\right\rangle \\
& =\left\langle-\langle I \vec{y}(x-\beta), \vec{u}\rangle \alpha^{\prime}(\vec{u})+\vec{Y}(x-\beta), \frac{\partial}{\partial t} \vec{u}(x, t)\right\rangle .
\end{aligned}
$$

Since $d \vec{u} / d t=J E^{\prime}(\vec{u})$, where $J=(\partial / \partial x) I^{-1}$ and $(\partial / \partial x) \vec{Y}=$ $I \vec{y}$, it follows that

$$
\begin{aligned}
\frac{d A}{d t}= & \left\langle\langle I \vec{y}(x-\beta(t)), \vec{u}\rangle \frac{\partial}{\partial x} \alpha^{\prime}(\vec{u})\right. \\
& \left.-I \vec{y}(x-\beta(t)), I^{-1} E^{\prime}(\vec{u})\right\rangle \\
= & \left\langle\langle I \vec{y}(x-\beta(t)), \vec{u}\rangle \frac{\partial}{\partial x} I^{-1} \alpha^{\prime}(\vec{u})\right. \\
& \left.-\vec{y}(x-\beta(t)), E^{\prime}(\vec{u})\right\rangle \\
= & -\left\langle B(\vec{u}), E^{\prime}(\vec{u})\right\rangle .
\end{aligned}
$$

Since $0<E\left(\vec{\varphi}_{0}\right)-E\left(\vec{u}_{0}\right)=E\left(\vec{\varphi}_{c}\right)-E(\vec{u}(t))$, from Lemma 24, we can deduce that

$$
0<\Pi(\vec{u}(t))\left\langle B(\vec{u}(t)), E^{\prime}(\vec{u}(t))\right\rangle .
$$

Since $\left\langle E^{\prime}\left(\vec{u}_{0}\right), B\left(\vec{u}_{0}\right)\right\rangle>0$ and it is continuous, we can obtain $\left\langle E^{\prime}(\vec{u}(t)), B(\vec{u}(t))\right\rangle>0$. Therefore for all $0<t<t_{1}, \Pi(\vec{u}(t))>$ 0 . Moreover, since $\vec{u}(t) \in U_{\varepsilon}$ and $\Pi\left(\vec{\varphi}_{c}\right)=0$, we may assume that $0<\Pi(\vec{u}(t))<1,0<t<t_{1}$, by choosing $\varepsilon$ smaller if necessary. So for all $t \in\left[0, t_{1}\right)$, by Lemma 24 , we have

$$
\begin{gathered}
\left\langle B(\vec{u}(t)), E^{\prime}(\vec{u})\right\rangle \\
\geq \Pi(\vec{u}(t))\left\langle B(\vec{u}(t)), E^{\prime}(\vec{u}(t))\right\rangle \\
>E\left(\vec{\varphi}_{c}\right)-E(\vec{u}(t)) \\
=E\left(\vec{\varphi}_{c}\right)-E\left(\vec{u}_{0}\right)=\varepsilon_{0}>0 \\
-\frac{d A}{d t} \geq E\left(\vec{\varphi}_{c}\right)-E\left(\vec{u}_{0}\right)=\varepsilon_{0}>0, \quad \text { for } t \in\left[0, t_{1}\right) .
\end{gathered}
$$

Integrating (126) on $\left[0, t_{1}\right)$, we have

$$
A(0)-A\left(t_{1}\right) \geq \varepsilon_{0} t_{1}=\left[E\left(\vec{\varphi}_{c}\right)-E\left(\vec{u}_{0}\right)\right] t_{1} .
$$

And then

$$
t_{1} \leq \frac{A(0)-A\left(t_{1}\right)}{E\left(\vec{\varphi}_{c}\right)-E\left(\vec{u}_{0}\right)} \leq \frac{2|A(t)|}{E\left(\vec{\varphi}_{c}\right)-E\left(\vec{u}_{0}\right)} .
$$

Since $|A(t)| \leq c_{2}\left(1+t^{2 / 3}+t^{9 / 10}\right)$, we can conclude that $t_{1}<\infty$. This completes the proof of Theorem 26.

\section{Conclusions}

In this paper, we studied the orbital stability and instability of solitary waves for (4) with two nonlinear terms. By using the orbital stability theory proposed in $[10,11]$, we obtained a general theorem judging the orbital stability for solitary waves of (4) in Section 3 based on proof of the local existence of the solutions, existence of the bounded state solution, and the spectral analysis of operator $H_{c}$. In Section 4, we gave the explicit expressions for the discrimination $d_{i}^{\prime \prime}(c), i=$ 1,2 , of orbital stability in terms of the two exact solitary waves $\left(\varphi_{i}, \psi_{i}\right)^{T}, i=1,2$, of (4). Furthermore, we deduced Theorems 9 and 10 which could easily judge the orbital stability of the two solitary waves $\left(\varphi_{i}, \psi_{i}\right)^{T}, i=1,2$, and analyzed the influence of the two nonlinear terms on the orbital stability. Finally, we studied instability in Section 5. We defined a new conservational functional and estimated to the solution of initial value problem to overcome the difficulty that we could not apply Grillakis-Shatah-Strauss theory on the system directly since $J$ is not onto. We constructed a formal Lyapunov function and proved Theorem 26.

\section{Conflict of Interests}

The authors declare that there is no conflict of interests regarding the publication of this paper.

\section{Acknowledgments}

This research is supported by the National Natural Science Foundation of China (Grant no. 11071164), Innovation Program of Shanghai Municipal Education Commission (Grant no. 13ZZ118), and Shanghai Leading Academic Discipline Project (Grant no. XTKX2012). 


\section{References}

[1] C. E. Seyler and D. L. Fenstermacher, "A symmetric regularizedlong-wave equation," Physics of Fluids, vol. 27, no. 1, pp. 4-7, 1984.

[2] I. L. Bogolubsky, "Some examples of inelastic soliton interaction," Computer Physics Communications, vol. 13, no. 3, pp. 149$155,1977$.

[3] B. L. Guo, "The spectral method for symmetric regularized wave equations," Journal of Computational Mathematics, vol. 5, no. 4, pp. 297-306, 1987.

[4] B. L. Guo, "The existence of global solution and "blow up" phenomenon for a system of multi-dimensional symmetric regularized wave equations," Acta Mathematicae Applicatae Sinica, vol. 8, no. 1, pp. 59-72, 1992.

[5] J. D. Zheng, R. F. Zhang, and B. Y. Guo, "The Fourier pseudospectral method for the SRLW equation," Applied Mathematics and Mechanics, vol. 10, no. 9, pp. 801-810, 1989 (Chinese).

[6] W.-G. Zhang, "Explicit exact solitary wave solutions for generalized symmetric regularized long-wave equations with highorder nonlinear terms," Chinese Physics, vol. 12, no. 2, pp. 144148, 2003.

[7] L. Chen, "Stability and instability of solitary waves for generalized symmetric regularized-long-wave equations," Physica D, vol. 118, no. 1-2, pp. 53-68, 1998.

[8] Y. B. Li, G. Q. Qin, and Z. H. Wang, Semigroups of Bounded Linear Operators and Applications, Liaoning Scientific and Technical Publishers, 1992 (Chinese).

[9] A. Pazy, Semigroups of Linear Operators and Applications to Partial Differential Equations, Springer, New York, NY, USA, 1983.

[10] M. Grillakis, J. Shatah, and W. Strauss, "Stability theory of solitary waves in the presence of symmetry. I," Journal of Functional Analysis, vol. 74, no. 1, pp. 160-197, 1987.

[11] M. Grillakis, J. Shatah, and W. Strauss, "Stability theory of solitary waves in the presence of symmetry. II," Journal of Functional Analysis, vol. 94, no. 2, pp. 308-348, 1990.

[12] E. M. Stein, Harmonic Analysis, Princeton University Press, Princeton, NJ, USA, 1993.

[13] Y. Liu, "Instability of solitary waves for generalized Boussinesq equations," Journal of Dynamics and Differential Equations, vol. 5, no. 3, pp. 537-558, 1993.

[14] J. L. Bona, P. E. Souganidis, and W. A. Strauss, "Stability and instability of solitary waves of Korteweg-de Vries type," Proceedings of the Royal Society of London A, vol. 411, no. 1841, pp. 395-412, 1987. 


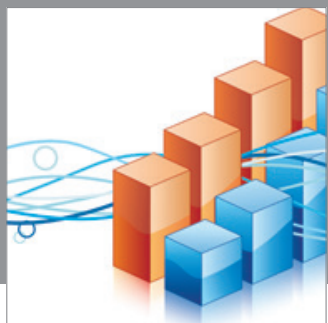

Advances in

Operations Research

mansans

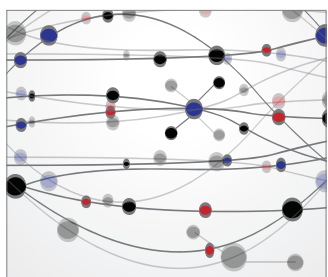

The Scientific World Journal
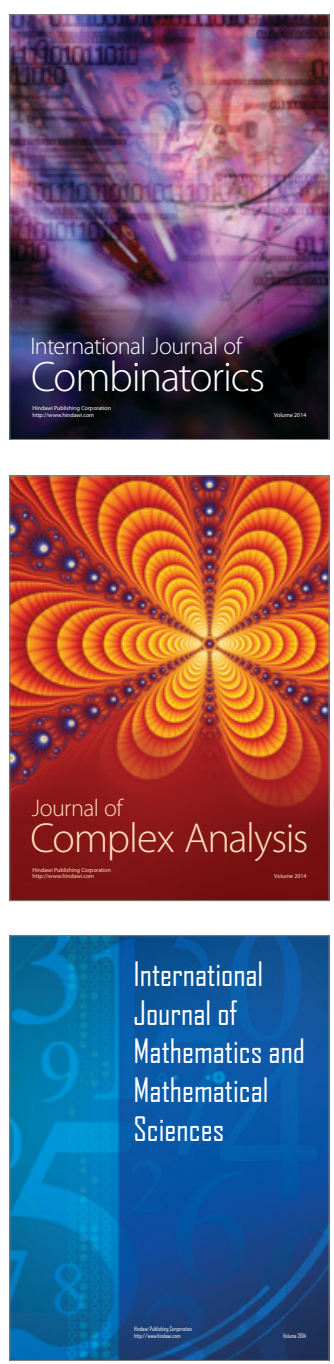
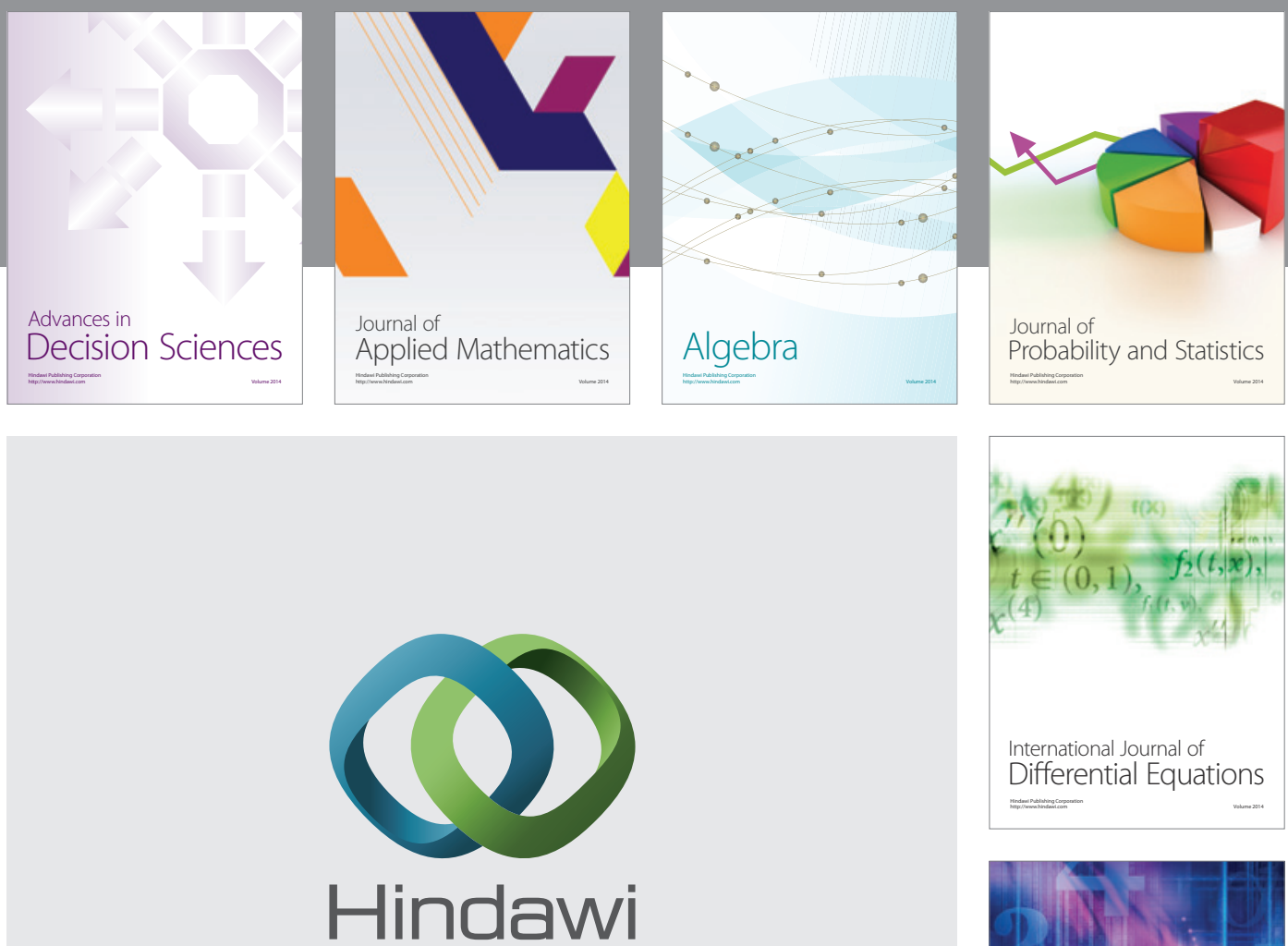

Submit your manuscripts at http://www.hindawi.com
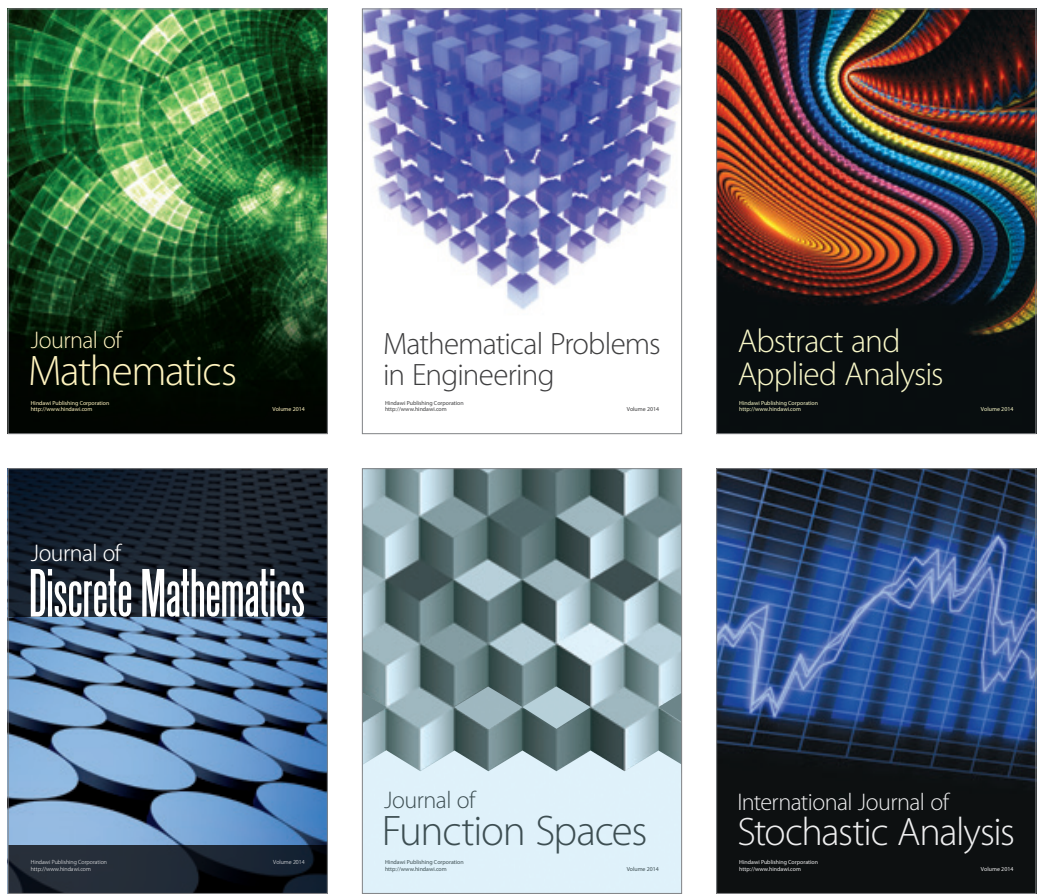

Journal of

Function Spaces

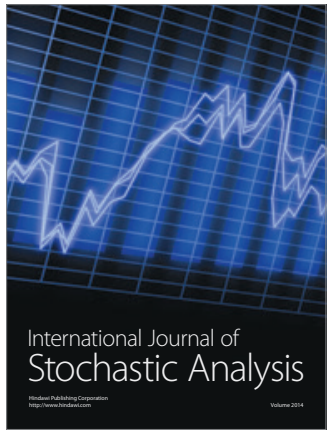

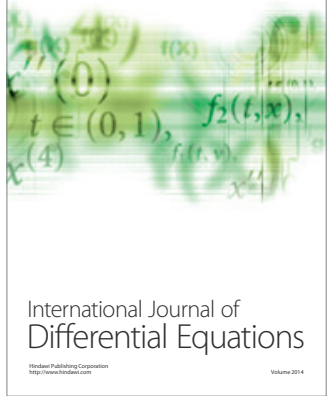
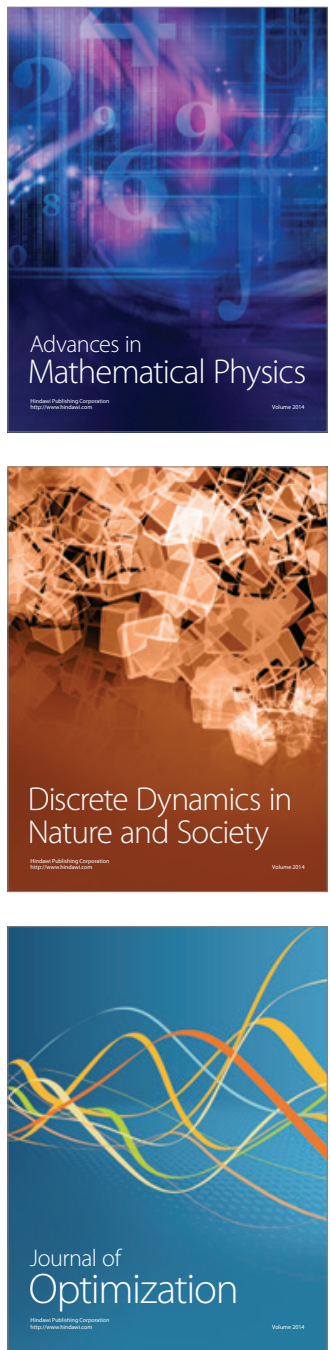